hep-th/9911107

SBNC/99-11-01

\title{
Renormalizability of the Dynamical Two-Form
}

\author{
Amitabha Lahiri \\ S. N. Bose National Centre for Basic Sciences, \\ Block JD, Sector III, Salt Lake, Calcutta 700 091, INDIA \\ amitabha@boson.bose.res.in \\ (Physical Review D (to be published))
}

\begin{abstract}
A proof of renormalizability of the theory of the dynamical non-Abelian two-form is given using the Zinn-Justin equation. Two previously unknown symmetries of the quantum action, different from the BRST symmetry, are needed for the proof. One of these is a gauge fermion dependent nilpotent symmetry, while the other mixes different fields with the same transformation properties. The BRST symmetry itself is extended to include a shift transformation by use of an anticommuting constant. These three symmetries restrict the form of the quantum action up to arbitrary order in perturbation theory. The results show that it is possible to have a renormalizable theory of massive vector bosons in four dimensions without a residual Higgs boson.
\end{abstract}

PACS 11.10.Gh, 11.30.Ly 


\section{INTRODUCTION}

Each and every aspect of the Standard Model has been tested in recent years, with remarkable agreement with theory, except in one sector. The Standard Model predicts the existence of the Higgs boson, responsible for making gauge bosons and fermions massive, as well as breaking the $\mathrm{SU}(2) \times \mathrm{U}(1)$ symmetry of the theory down to the $\mathrm{U}(1)$ of electromagnetism. But no elementary scalar has yet been observed in any particle interaction, nor has any experiment so far detected the Higgs boson, either elementary or composite. On the other hand, various theoretical constraints put the upper bound of the Higgs boson mass only a little out of reach of present day experiments. It is therefore useful to consider the scenario in which the Higgs boson remains unobserved as the theoretical bounds are reached.

Apart from the Higgs boson and a possible neutrino mass, the Standard Model agrees quite closely with experiment, so it is a good idea to leave most of the theory untouched. The role of the Higgs boson may be distributed among possibly different mechanisms for generating vector and fermion masses, and symmetry breaking. The Higgs mechanism does all this in a renormalizable and unitary way [1], and any alternative must not affect these good quantum properties of the theory. A possible alternative for generating vector boson masses is to use a dynamical two-form. When an antisymmetric tensor potential $B$ is coupled to the field strength $F$ of a U(1) gauge field via a 'topological' $B \wedge F$ coupling and a kinetic term for $B$ is included, the gauge field develops an effective mass [2 2 [5]. The mass is equal to the dimensionful coupling constant $m$ of the interaction term, and there is no residual scalar (Higgs) degree of freedom. If a non-Abelian version of this theory can be consistently quantized, it may be applied to particle interactions.

No-go theorems [6] [0] based on the consistency of quantum symmetries rule out most, but not all, alternative Higgs free mechanisms of mass generation for non-Abelian vector bosons. One useful exception is the topological mass generation mechanism [8] which has seen renewed interest in recent years [9 11]. This mechanism uses an auxiliary vector field to close the symmetry algebra and thus avoid the no-go theorems. The price one has to pay is to have non-propagating bosonic and ghost fields in the theory, which disappear in the Abelian limit. The no-go theorem of Ref. [7] says only that the non-Abelian model cannot be constructed from the Abelian model, which is known to be quantizable [4]. It does not rule out the quantizability of the non-Abelian model itself. However that is not in itself a proof that the non-Abelian model is quantizable, and a proof has not been constructed as yet. The first step in such a proof is the construction of a BRST-invariant tree-level action, which was done from a geometric point of view in [9] and ab initio in [10].

In this paper I construct the quantum action for this model up to arbitrary order in perturbation theory starting from the BRST-invariant tree-level action. I follow an algebraic procedure along the lines of what is done for Yang-Mills theories [12,13]. The construction itself is rather involved as there are different fields with the same transformation properties. This suggests that the usual BRST symmetry is not sufficient to restrict the operators in the quantum action. Fortunately there are other useful symmetries of the tree-level action and they, together with the BRST symmetry, are sufficient for the purpose. The starting point of the paper is the classical action given in Sec. [1]. In Sec. III I list the BRST transformation rules of the theory and construct another BRST-like nilpotent symmetry. In Sec. IV I construct the quantum symmetries corresponding to these and other symmetries, 
and in Sec. $\mathrm{V}$ I find all the dimension four operators allowed by all the symmetries. Finally, Sec. VI carries a small discussion of possible extensions and applications of the results. The main body of the paper sets up the structure of the proof, most of the detailed calculations are collected in appendices at the end.

\section{TREE LEVEL ACTION}

In this section I shall fix my conventions. I shall work with an $\mathrm{SU}(\mathrm{N})$ gauge group $G$, with generators $t_{a}$ satisfying

$$
\left[t_{a}, t_{b}\right]=i f^{a b c} t_{c}
$$

with the structure constants $f^{a b c}$ totally antisymmetric in its indices. The gauge index, as well as Lorentz indices, will be made explicit in general for easier tracking of numerical coefficients. The background metric is taken to have signature $(-+++)$.

The classical action for the dynamical non-Abelian two-form [8] is

$$
S_{0}=\int d^{4} x\left(-\frac{1}{4} F_{\mu \nu}^{a} F^{a \mu \nu}-\frac{1}{12} H_{\mu \nu \lambda}^{a} H^{a \mu \nu \lambda}+\frac{m}{4} \epsilon^{\mu \nu \rho \lambda} B_{\mu \nu}^{a} F_{\rho \lambda}^{a}\right) .
$$

Here $F_{\mu \nu}$ is the curvature of a gauge connection $A_{\mu}$ with gauge coupling $g$,

$$
F_{\mu \nu}^{a}=\left(\frac{i}{g}\left[D_{\mu}, D_{\nu}\right]\right)^{a}=\partial_{\mu} A_{\nu}^{a}-\partial_{\nu} A_{\mu}^{a}+g f^{a b c} A_{\mu}^{b} A_{\nu}^{c} .
$$

The compensated field strength $H_{\mu \nu \lambda}$ is defined with the help of an auxiliary field $C_{\mu}$ by the relation

$$
\begin{aligned}
H_{\mu \nu \lambda}^{a} & =\left(D_{[\mu} B_{\nu \lambda]}\right)^{a}+i g\left[F_{[\mu \nu}, C_{\lambda]}\right]^{a} \\
& =\partial_{[\mu} B_{\nu \lambda]}^{a}+g f^{a b c} A_{[\mu}^{b} B_{\nu \lambda]}^{c}-g f^{a b c} F_{[\mu \nu}^{b} C_{\lambda]}^{c} .
\end{aligned}
$$

All the three fields $A_{\mu}, B_{\mu \nu}$ and $C_{\mu}$ belong to the adjoint representation of the gauge group $G$. The action (2.2) therefore remains invariant under gauge transformations given by

$$
A_{\mu} \rightarrow U A_{\mu} U^{-1}-\frac{i}{g} \partial_{\mu} U U^{-1}, \quad B_{\mu \nu} \rightarrow U B_{\mu \nu} U^{-1}, \quad C_{\mu} \rightarrow U C_{\mu} U^{-1}, \quad U \in G .
$$

In addition, the action $S_{0}$ is invariant under vector gauge transformations given by

$$
A_{\mu} \rightarrow A_{\mu}, \quad B_{\mu \nu} \rightarrow B_{\mu \nu}+D_{[\mu} \Lambda_{\nu]}, \quad C_{\mu} \rightarrow C_{\mu}+\Lambda_{\mu}
$$

where $\Lambda_{\mu}$ is some arbitrary vector field in the adjoint representation of the gauge group which vanishes at infinity.

For the purpose of power counting, I need the propagators of this theory. Let me choose the usual Lorenz gauge $\partial_{\mu} A^{a \mu}=0, \partial_{\nu} B^{a \mu \nu}=0$, with gauge parameters $\xi$ and $\eta$ respectively. Then the tree-level propagator for $A_{\mu}^{a}$ is

$$
D_{\mu \nu}^{a b}=-\frac{\delta^{a b}}{k^{2}}\left(g_{\mu \nu}-(1-\xi) \frac{k_{\mu} k_{\nu}}{k^{2}}\right),
$$


and that for $B_{\mu \nu}^{a}$ is

$$
D_{\mu \nu, \rho \lambda}^{a b}=-\frac{\delta^{a b}}{k^{2}}\left(g_{\mu[\rho} g_{\lambda] \nu}-(1-\eta) \frac{g_{\mu[\rho} k_{\lambda]} k_{\nu}}{k^{2}}\right) .
$$

There is no quadratic term in the action involving the auxiliary field $C_{\mu}^{a}$ in this gauge, so the tree level propagator for it will vanish. As a result, there are no diagrams with internal $C_{\mu}^{a}$ lines. This may look very peculiar, but it is also possible to choose some other gauge for which $C_{\mu}^{a}$ has a non-vanishing (gauge-dependent) propagator, but that does not change the arguments. This is discussed in the last section. For the moment let me proceed without a quadratic term for the auxiliary field $C_{\mu}^{a}$.

There is a quadratic coupling term between the vector and the antisymmetric tensor fields coming from the last term in the action, the vertex given by

$$
V_{\nu, \rho \lambda}^{a b}=i m \delta^{a b} \epsilon_{\mu \nu \rho \lambda} k^{\mu} .
$$

The effective tree-level propagator for the vector field is then calculated by summing all insertions of the tree-level $B_{\mu \nu}$ propagator into the naïve tree-level $A_{\mu}$ propagator [4]. The result is

$$
\widetilde{D}_{\mu \nu}^{a b}=-\frac{\delta^{a b}}{k^{2}-m^{2}}\left(g_{\mu \nu}-\frac{k_{\mu} k_{\nu}}{k^{2}}\right)-\delta^{a b} \xi \frac{k_{\mu} k_{\nu}}{k^{4}} .
$$

This shows that there is a pole in the two-point function of the vector field $A_{\mu}^{a}$ even at tree-

level. On the other hand, the 'massive' propagator $\widetilde{D}_{\mu \nu}^{a b}$ falls off as $1 / k^{2}$ at high values of $k^{2}$, like in the case of the Higgs mechanism. The ultraviolet behavior of the propagators show that the theory is power-counting renormalizable in this gauge. The best way to proceed further is via the BRST method of quantization.

\section{BRST INVARIANCE}

Quantization of this theory requires gauge-fixing and therefore the introduction of ghosts. The gauge fixed action, together with the ghost terms, is BRST invariant. The vector gauge symmetry requires ghosts of ghosts, and off-shell nilpotence of the BRST charge requires auxiliary fields. Let me write the gauge-fixing functions as $f^{a}, f^{a \mu}$ and $f^{\prime a}$ for gauge transformations, vector gauge transformations and gauge transformations of ghosts, respectively. In Sec. \, I shall choose the gauge functions to be of the usual Lorenz gauge type,

$$
f^{a}=\partial_{\mu} A^{a \mu}, \quad f^{a \mu}=\partial_{\nu} B^{a \mu \nu}, \quad f^{\prime a}=\partial_{\mu} \omega^{a \mu},
$$

but most of the results in this paper will hold for arbitrary linear gauge functions. Some discussion about arbitrary gauges is presented in Sec. VI.

The tree level quantum action can be written as

$$
\begin{aligned}
S=S_{0}+\int d^{4} x & {\left[h^{a} f^{a}+\bar{\omega}^{a} \Delta^{a}+\frac{1}{2} \xi h^{a} h^{a}+h_{\mu}^{a}\left(f^{a \mu}+\partial^{\mu} n^{a}\right)\right.} \\
+ & \left.\bar{\omega}_{\mu}^{a} \Delta^{a \mu}+\frac{1}{2} \eta h_{\mu}^{a} h^{a \mu}-\partial_{\mu} \bar{\omega}^{a \mu} \alpha^{a}+\bar{\alpha}^{a} f^{\prime a}+\bar{\beta}^{a} \Delta^{\prime a}+\zeta \bar{\alpha}^{a} \alpha^{a}\right] .
\end{aligned}
$$


Here $\Delta^{a}, \Delta^{a \mu}$ and $\Delta^{\prime a}$ are the BRST variations, as defined below, of $f^{a}, f^{a \mu}$ and $f^{\prime a}$, respectively. The appearance of $\partial_{\mu} n^{a}$ in the gauge-fixing condition is usual for two-form gauge-fields. The gauge-fixing condition $f^{a \mu}=0$ holds upon using the equation of motion of $n^{a}$ [15]. This action is no longer invariant under gauge or vector gauge transformations. But it is invariant under the BRST transformations [9, 10]

$$
\begin{aligned}
& s A_{\mu}^{a}=\partial_{\mu} \omega^{a}+g f^{a b c} A_{\mu}^{b} \omega^{c}, \\
& s \omega^{a}=-\frac{1}{2} g f^{a b c} \omega^{b} \omega^{c}, \quad s \bar{\omega}^{a}=-h^{a}, \quad s h^{a}=0, \\
& s B_{\mu \nu}^{a}=g f^{a b c} B_{\mu \nu}^{b} \omega^{c}+\left(D_{[\mu} \omega_{\nu]}\right)^{a}+g f^{a b c} F_{\mu \nu}^{b} \theta^{c}, \\
& s C_{\mu}^{a}=g f^{a b c} C_{\mu}^{b} \omega^{c}+\omega_{\mu}^{a}+\left(D_{\mu} \theta\right)^{a}, \\
& s \omega_{\mu}^{a}=-g f^{a b c} \omega_{\mu}^{b} \omega^{c}+\left(D_{\mu} \beta\right)^{a}, \\
& s \bar{\omega}_{\mu}^{a}=-h_{\mu}^{a}, \quad s h_{\mu}^{a}=0, \quad s n^{a}=\alpha^{a}, \quad s \alpha^{a}=0, \\
& s \beta^{a}=g f^{a b c} \beta^{b} \omega^{c}, \\
& s \bar{\beta}^{a}=\bar{\alpha}^{a}, \quad s \bar{\alpha}^{a}=0, \\
& s \theta^{a}=-g f^{a b c} \theta^{b} \omega^{c}-\beta^{a} .
\end{aligned}
$$

These transformations are nilpotent, $s^{2}=0$ on all fields, if $s$ has a left action, i.e., the change in any field $\chi^{A}$ is given by $\delta \chi^{A}=\delta \lambda s \chi^{A}$, where $\delta \lambda$ is an anticommuting infinitesimal parameter. The tree-level quantum action of Eqn.(3.2) is invariant under $s$, with $\Delta^{a}=s f^{a}$, $\Delta^{a \mu}=s f^{a \mu}$ and $\Delta^{\prime a}=s f^{\prime a}$. It is also possible to write this action as the sum of the classical action $S_{0}$ plus a total super-divergence,

$$
\begin{gathered}
S=S_{0}+s \Psi, \\
\Psi=-\left(\bar{\omega}^{a} f^{a}+\frac{1}{2} \xi \bar{\omega}^{a} h^{a}\right)-\left(\bar{\omega}_{\mu}^{a}\left(f^{a \mu}+\partial^{\mu} n^{a}\right)+\frac{1}{2} \eta \bar{\omega}_{\mu}^{a} h^{a \mu}\right)+\left(\bar{\beta}^{a} f^{\prime a}+\zeta \bar{\beta}^{a} \alpha^{a}\right) .
\end{gathered}
$$

In addition to the BRST transformations, there is another BRST-type nilpotent transformation which leaves the action invariant. Such a symmetry exists for all gauge theories, not just the two-form theories, as can be seen from the following argument. The terms in the extended ghost sector of the tree-level quantum action of a gauge theory are typically of the form

$$
S_{e x t}^{c}=h^{A} f^{A}+\frac{1}{2} \lambda h^{A} h^{A}+\bar{\omega}^{A} \Delta^{A}
$$

where $\left(\bar{\omega}^{A}, h^{A}\right)$ are the trivial pairs. Here the index $A$ stands for the collection of various indices as well as the space-time point where the fields are evaluated, $f^{A}=0$ is the corresponding gauge-fixing condition with gauge parameter $\lambda$, and $\Delta^{A}=s f^{A}$. The sum over $A$ includes the integration over space-time. This form of the extended ghost sector is valid for commuting $h^{A}, f^{A}$ and anticommuting $\bar{\omega}^{A}$. For example, all but the last three terms of the tree-level quantum action (3.2) can be written in this form, where the index $A$ includes the gauge index $a$ or the pair $(a, \mu)$ depending on the gauge field $\left(A_{\mu}\right.$ or $\left.B_{\mu \nu}\right)$. This part of the action remains invariant under BRST transformations

$$
s \bar{\omega}^{A}=-h^{A}, \quad s h^{A}=0 .
$$


On the other hand, I can rearrange $S_{e x t}^{c}$ as

$$
\begin{aligned}
S_{e x t}^{c} & =\frac{1}{2} \lambda\left(h^{A}+\frac{1}{\lambda} f^{A}\right)\left(h^{A}+\frac{1}{\lambda} f^{A}\right)-\frac{1}{2 \lambda} f^{A} f^{A}+\bar{\omega}^{A} \Delta^{A} \\
& =\frac{1}{2} \lambda\left(\left(h^{A}+\frac{2}{\lambda} f^{A}\right)-\frac{1}{\lambda} f^{A}\right)\left(\left(h^{A}+\frac{2}{\lambda} f^{A}\right)-\frac{1}{\lambda} f^{A}\right)-\frac{1}{2 \lambda} f^{A} f^{A}+\bar{\omega}^{A} \Delta^{A} \\
& =\frac{1}{2} \lambda\left(h^{\prime A}+\frac{1}{\lambda} f^{A}\right)\left(h^{\prime A}+\frac{1}{\lambda} f^{A}\right)-\frac{1}{2 \lambda} f^{A} f^{A}+\bar{\omega}^{A} \Delta^{A} \\
& =h^{\prime A} f^{A}+\frac{1}{2} \lambda h^{\prime A} h^{\prime A}+\bar{\omega}^{A} \Delta^{A},
\end{aligned}
$$

where I have defined $h^{\prime A}=-h^{A}-\frac{2}{\lambda} f^{A}$. So far, I have not actually done anything. The only thing that comes out of this exercise is the fact that $S_{e x t}^{c}$ is invariant under a new set of BRST transformations:

$$
\begin{gathered}
\tilde{s} \bar{\omega}^{A}=-h^{\prime A} \Rightarrow \tilde{s} \bar{\omega}^{A}=h^{A}+\frac{2}{\lambda} f^{A}, \\
\tilde{s} h^{\prime A}=0 \Rightarrow \tilde{s} h^{A}=-\frac{2}{\lambda} \tilde{s} f^{A}, \\
\tilde{s} f^{A}=\Delta^{A} \equiv s f^{A} .
\end{gathered}
$$

Therefore, if the action of $\tilde{s}$ on $\bar{\omega}^{A}$ and $h^{A}$ is as above, and $\tilde{s}=s$ on all other fields, the last equation is identically satisfied, and it also follows that $\tilde{s}$ is nilpotent on all fields, $\tilde{s}^{2}=0$ if $\Delta^{A}$ does not contain any auxiliary field, which is usually the case.

When the extended sector corresponds to an anticommuting gauge field, as in the case of gauge-fixing of ghost fields, the construction is slightly more complicated, since the auxiliary fields have odd ghost number. Typically, for anticommuting auxiliary fields $\bar{\alpha}^{A}, \alpha^{A}$, the extended ghost sector can be written as

$$
S_{e x t}^{a}=\bar{\alpha}^{A} f^{\prime A}+\bar{f}^{\prime A} \alpha^{A}+\zeta \bar{\alpha}^{A} \alpha^{A}+\bar{\beta}^{A} \Delta^{\prime A}
$$

In this, $f^{\prime A}$ is the anticommuting gauge-fixing function, $\Delta^{\prime A}=s f^{\prime A}$, and $\bar{\beta}^{A}$ is the corresponding commuting antighost. The term $\bar{f}^{\prime A} \alpha^{A}$ is just a rearrangement of the appropriate terms in $\bar{\omega}^{A} \Delta^{A}$ which appear for the usual gauge symmetries. A term such as $\bar{f}^{\prime A} \alpha^{A}$ must appear, since $\alpha^{A}$ is the ghost for some field and therefore appears in some $\Delta^{A}$. For example, in the tree-level quantum action of Eqn.(3.2) $\bar{f}^{\prime A} \alpha^{A}$ corresponds to $\bar{\omega}^{a \mu} \partial_{\mu} \alpha^{a}$, which in turn is required to cancel the BRST variation of $h_{\mu}^{a} \partial^{\mu} n^{a}$. Just as in the case with commuting auxiliary fields, the terms in $S_{e x t}^{a}$ can be rearranged,

$$
\begin{aligned}
S_{e x t}^{a} & =\zeta\left(\bar{\alpha}^{A}+\frac{1}{\zeta} \bar{f}^{\prime A}\right)\left(\alpha^{A}+\frac{1}{\zeta} f^{\prime A}\right)-\frac{1}{\zeta} \bar{f}^{\prime A} f^{A}+\bar{\beta}^{A} \Delta^{\prime A} \\
& =\zeta\left(\left(\bar{\alpha}^{A}+\frac{2}{\zeta} \bar{f}^{\prime A}\right)-\frac{1}{\zeta} \bar{f}^{\prime A}\right)\left(\left(\alpha^{A}+\frac{2}{\zeta} f^{\prime A}\right)-\frac{1}{\zeta} f^{\prime A}\right)-\frac{1}{\zeta} \bar{f}^{\prime A} f^{A}+\bar{\beta}^{A} \Delta^{\prime A} \\
& =\zeta\left(\bar{\alpha}^{\prime A}+\frac{1}{\zeta} \bar{f}^{\prime A}\right)\left(\alpha^{\prime A}+\frac{1}{\zeta} f^{\prime A}\right)-\frac{1}{\zeta} \bar{f}^{\prime A} f^{A}+\bar{\beta}^{A} \Delta^{\prime A} \\
& =\zeta \bar{\alpha}^{\prime A} \alpha^{\prime A}+\bar{\alpha}^{\prime A} f^{\prime A}+\bar{f}^{\prime A} \alpha^{\prime A}+\bar{\beta}^{A} \Delta^{\prime A} .
\end{aligned}
$$


where I have now defined $\bar{\alpha}^{\prime A}=-\left(\bar{\alpha}^{A}+\frac{2}{\zeta} \bar{f}^{\prime A}\right)$ and $\alpha^{\prime A}=-\left(\alpha^{A}+\frac{2}{\zeta} f^{\prime A}\right)$. As before, in these coordinates $S_{\text {ext }}^{a}$ is invariant under its own set of BRST transformations,

$$
\begin{array}{r}
\tilde{s} \bar{\beta}^{A}=\bar{\alpha}^{\prime A}=-\left(\bar{\alpha}^{A}+\frac{2}{\zeta} \bar{f}^{\prime A}\right), \\
\tilde{s} \bar{\alpha}^{\prime A}=0 \Rightarrow \tilde{s} \bar{\alpha}^{A}=-\frac{2}{\zeta} \tilde{s} \bar{f}^{\prime A}, \\
\tilde{s} \alpha^{\prime A}=0 \Rightarrow \tilde{s} \alpha^{A}=-\frac{2}{\zeta} \tilde{s} f^{\prime A}, \\
\tilde{s} f^{\prime A}=\Delta^{\prime A} \equiv s f^{\prime A} .
\end{array}
$$

Two more things are required for the nilpotence of $\tilde{s}-\alpha^{A}$ was the result of BRST variation of some field $\left(\alpha^{a}=s n^{a}\right.$ in Eqn.(3.3) $)$ - now $\alpha^{\prime A}$ has to be the variation under $\tilde{s}$ of the same field, and $\tilde{s} \bar{f}^{\prime A}$ must be calculated according to the rules of Eqn.(3.8) for $\tilde{s}$ acting on the anticommuting ghosts in $\bar{f}^{\prime A}$. In addition, the action of $\tilde{s}$ must be the same as that of $s$ for the fields contained in $f^{\prime A}$. Then $\tilde{s}^{2}=0$ on all fields.

I can now gather the results of Eqn.(3.8) and Eqn.(3.11) and apply them to the tree-level quantum action of Eqn.(3.2) to construct this symmetry,

$$
\begin{aligned}
\tilde{s} \bar{\omega}^{a} & =h^{a}+\frac{2}{\xi} f^{a}, \\
\tilde{s} h^{a} & =-\frac{2}{\xi} \Delta^{a}, \\
\tilde{s} \bar{\omega}_{\mu}^{a} & =h_{\mu}^{a}+\frac{2}{\eta} \partial_{\mu} n^{a}+\frac{2}{\eta} f_{\mu}^{a}, \\
\tilde{s} h_{\mu}^{a} & =\frac{2}{\eta}\left(\partial_{\mu} \alpha^{a}+\frac{2}{\zeta} \partial_{\mu} f^{\prime a}-\Delta_{\mu}^{a}\right), \\
\tilde{s} n^{a} & =-\left(\alpha^{a}+\frac{2}{\zeta} f^{\prime a}\right) \\
\tilde{s} \bar{\beta}^{a} & =-\left(\bar{\alpha}^{a}-\frac{2}{\zeta} \partial_{\mu} \bar{\omega}^{a \mu}\right), \\
\tilde{s} \alpha^{a} & =-\frac{2}{\zeta} \Delta^{\prime a}, \\
\tilde{s} \bar{\alpha}^{a} & =\frac{2}{\zeta} \tilde{s}\left(\partial_{\mu} \bar{\omega}^{a \mu}\right)=\frac{2}{\zeta} \partial_{\mu}\left(h^{a \mu}+\frac{2}{\eta} \partial^{\mu} n^{a}+\frac{2}{\eta} f^{a \mu}\right), \\
\tilde{s} & =s \text { on all other fields. }
\end{aligned}
$$

Since the gauge-fixing functions do not contain antighosts or auxiliary fields, and since BRST variations of the remaining fields also do not contain antighosts or auxiliary fields, a straightforward calculation shows that $\tilde{s}$ is nilpotent on all fields,

$$
\tilde{s}^{2}=0 \text {. }
$$

In addition, since the classical action $S_{0}$ is invariant under BRST transformations, and since $\tilde{s}=s$ on the fundamental fields, 


$$
\tilde{s} S_{0}=0
$$

The remainder of the tree-level quantum action of Eqn. (3.2) can be written as a sum of $S_{e x t}^{c}$ and $S_{\text {ext }}^{a}$ as defined above, and either by the method described above or by an explicit calculation it can be shown quite easily that this part is also invariant under $\tilde{s}$. So in fact

$$
\tilde{s} S=0 \text {. }
$$

It should be made clear that $\tilde{s}$ is not special to the dynamical two-form, nor even to reducible gauge systems. Usual gauge theories exhibit invariance under a symmetry analogous to $\tilde{s}$. But in those cases, this gauge-fermion dependent invariance is not needed for restricting the form of the quantum action - invariance under the familiar BRST transformation $s$ is sufficient for that purpose [12,13. However, $\tilde{s}$ becomes extremely useful when the theory contains many different fields in the same representation, as in the case of the dynamical two-form. I shall make extensive use of $\tilde{s}$ to construct the quantum effective action for the dynamical two-form. In order to do that, I need to look at the quantum symmetries corresponding to $s, \tilde{s}$ and some other classical symmetries of the theory. This is done in the next section.

\section{SYMMETRIES OF THE EFFECTIVE ACTION}

On the way to a proof of perturbative renormalizability of the dynamical non-Abelian two-form, the first thing to note is that there is no kinetic term for $C_{\mu}^{a}$ in the tree-level action. Consequently, $C_{\mu}^{a}$ is taken to be dimensionless. The auxiliary ghost field $\theta$ is taken to be dimensionless for the same reason, and then the theory is power-counting renormalizable. The presence of fields with vanishing mass dimension does not automatically rule out renormalizability of a theory [14, but it is possible that the theory will be non-renormalizable because counterterms may contain arbitrary powers of these fields. Therefore, one needs to ensure that the symmetries of the theory restrict the number of counterterms to a finite value. Perturbative renormalizability requires that the quantum effective action, invariant under the quantum symmetries, contain only those operators which appear in the tree-level action of Eqn. (3.2) up to arbitrary numerical coefficients. The quantum action can be constructed by use of the Zinn-Justin equation in the following manner.

The partition function $Z[J, K]$ in the presence of external $c$-number sources $J^{A}(x), K^{A}(x)$ is

$$
Z[J, K]=\int\left[\mathscr{D} \chi^{B}\right] \exp \left(i S+i \int d^{4} x \chi^{A} J^{A}+i \int d^{4} x F^{A} K^{A}\right)
$$

where $F^{A}(x)=s \chi^{A}(x)$, and I have kept the space-time integration explicit for this section. I shall also refer to $K^{A}$ as the 'antisource' corresponding to the field $\chi^{A}$. This partition function leads to the effective action

$$
\Gamma[\chi, K]=-\int d^{4} x \chi^{A} J_{\chi, K}^{A}-i \ln Z\left[J_{\chi, K}, K\right],
$$

where $J_{\chi, K}^{A}$ is the value of the current for which $\left\langle\chi^{B}(x)\right\rangle_{J, K}=\chi^{B}(x)$, the expectation value being calculated in the presence of $K^{A}$. 
The effective action satisfies the Zinn-Justin equation [12,13],

$$
(\Gamma, \Gamma)=0,
$$

where the antibracket $(F, G)$ is defined for any two functionals $F$ and $G$ as

$$
(F, G)=\int d^{4} x \frac{\delta_{R} F[\chi, K]}{\delta \chi^{A}(x)} \frac{\delta_{L} G[\chi, K]}{\delta K^{A}(x)}-\int d^{4} x \frac{\delta_{R} F[\chi, K]}{\delta K^{A}(x)} \frac{\delta_{L} G[\chi, K]}{\delta \chi^{A}(x)} .
$$

In order to get a proof of perturbative renormalizability of a theory, the total action functional $S[\chi, K]=S[\chi]+\int d^{4} x F^{A} K^{A}$ is written as a sum of the renormalized action $S_{R}[\chi, K]$ plus a term $S_{\infty}[\chi, K]$ containing counterterms intended to cancel loop infinities. Both $S_{R}$ and $S_{\infty}$ must have the same symmetries as $S[\chi, K]$, so the infinite contributions to $\Gamma$ can be canceled by the counterterms in $S_{\infty}$ if they also have those symmetries.

Expanding $\Gamma$ in a power series in the loop expansion parameter $\hbar$,

$$
\Gamma[\chi, K]=\sum_{N=0}^{\infty} \hbar^{N-1} \Gamma_{N}[\chi, K],
$$

where $\Gamma_{0}[\chi, K]=S_{R}[\chi, K]$, the Zinn-Justin equation can be written order-by order for each $N$ as

$$
\sum_{N^{\prime}=0}^{N}\left(\Gamma_{N^{\prime}}, \Gamma_{N-N^{\prime}}\right)=0
$$

This expansion automatically includes counterterms corresponding to sub-divergences at any given loop order $N$. If for some $N$ all infinities appearing at $M$-loop order have been canceled by counterterms in $S_{\infty}$ for all $M \leq N-1$, the only remaining infinities in Eqn.(1.6) are in $\Gamma_{N}$. So the infinite part $\Gamma_{N, \infty}$ of this quantity must satisfy

$$
\left(S_{R}, \Gamma_{N, \infty}\right)=0 .
$$

For a theory which is renormalizable in the power-counting sense, this leads to a simple mechanical procedure. For such a theory, the infinite part $\Gamma_{N, \infty}[\chi, K]$ must be a sum of operators of mass dimension four or less. In addition, all the linear symmetries of the tree-level action are symmetries of $\Gamma[\chi, K]$ and therefore of $\Gamma_{N, \infty}[\chi, K]$.

Let me assume for the moment that $\Gamma_{N, \infty}[\chi, K]$ is at most linear in the antisources $K^{A}$ for all $A$,

$$
\Gamma_{N, \infty}[\chi, K]=\Gamma_{N, \infty}[\chi, 0]+\int d^{4} x \mathscr{F}_{N}^{A}[\chi, x] K^{A}(x) .
$$

If I now define the quantities

$$
\Gamma_{N}^{(\epsilon)}[\chi]=S_{R}[\chi, 0]+\epsilon \Gamma_{N, \infty}[\chi, 0],
$$

with $\epsilon$ infinitesimal, the terms independent of $K^{A}$ in Eqn.(4.7) imply [13] that $\Gamma_{N}^{(\epsilon)}[\chi]$ is invariant under the transformation

$$
s_{R} \chi^{A}(x)=F_{N}^{(\epsilon) A}(x),
$$


where

$$
F_{N}^{(\epsilon) A}(x)=F^{A}(x)+\epsilon \mathscr{F}_{N}^{A}(x) .
$$

The terms of first order in $K^{A}$ in Eqn.(4.7) imply that this transformation is nilpotent, $s_{R}^{2}=0$. Since $\Gamma_{N, \infty}$ contains only operators of mass dimension four or less, $F_{N}^{(\epsilon) A}(x)$ cannot be of higher mass dimension than $F^{A}(x)$. In addition, $F_{N}^{(\epsilon) A}(x)$ may not affect the linear symmetries of the action. Therefore, $F_{N}^{(\epsilon) A}(x)$ must have the same Lorentz properties, ghost number and global gauge transformation properties as $F^{A}(x)$. In fact $F_{N}^{(\epsilon) A}(x)$ must be the same as $F^{A}(x)$ if it corresponds to a field which transforms linearly under $s$. All that remains to be done is to construct the most general nilpotent transformation of the fields under these restrictions, and then to construct the most general functional $\Gamma_{N}^{(\epsilon)}[\chi]$ invariant under this transformation. If that agrees, up to arbitrary constant numerical coefficients, with the original action $S$, the theory is perturbatively renormalizable.

This entire argument rests on the assumption that $\Gamma_{N, \infty}[\chi, K]$ is at most linear in all of the antisources $K^{A}$. When is this a correct assumption? If a field $\chi^{A}$ has mass dimension $d_{A}$, the corresponding $K^{A}$ must have mass dimension $3-d_{A}$ so as to make $\int d^{4} x F^{A} K^{A}$ dimensionless. The antisources $K^{A}$ for $A_{\mu}^{a}, \omega^{a}, \bar{\omega}^{a}, B_{\mu \nu}^{a}, \omega_{\mu}^{a}, \bar{\omega}_{\mu}^{a}, n^{a}, \beta^{a}, \bar{\beta}^{a}$ all have mass dimension 2. The antisources for $C_{\mu}^{a}$ and $\theta^{a}$ each have mass dimension 3. Also, the theory does not have any external antisource $K^{A}$ for the fields $h^{a}, h_{\mu}^{a}, \alpha^{a}, \bar{\alpha}^{a}$ because their BRST variations vanish. Therefore $\Gamma_{N, \infty}$ can be at most quadratic in $K^{A}$.

If a field $\chi^{A}$ has ghost number $\gamma_{A}$, the corresponding $K^{A}$ will have ghost number $-\gamma_{A}-1$. It follows that the ghost number of the antisource for any of $A_{\mu}^{a}, B_{\mu \nu}^{a}, C_{\mu}^{a}, n^{a}$ is -1 . The ghost numbers of $K^{A}$ corresponding to $\omega^{a}, \omega_{\mu}^{a}$ and $\theta^{a}$ is -2 , and those of $K^{A}$ corresponding to $\beta^{a}$ and $\bar{\beta}^{a}$ are -3 and +1 , respectively. The remaining antisources correspond to $\bar{\omega}^{a}$ and $\bar{\omega}_{\mu}^{a}$, they carry ghost number 0 . The dimensions and ghost numbers of all the fields and their antisources are given in Table \at the end of this paper.

Some of the quadratic terms can be eliminated straightaway. The BRST variations of the fields $\bar{\omega}^{a}, \bar{\omega}_{\mu}^{a}, n^{a}$ and $\bar{\beta}^{a}$ are linear, so the effective action cannot be quadratic in their antisources. For example,

$$
s \bar{\omega}^{a}=-h^{a},
$$

so the quantum transformations are the same,

$$
\left\langle s \bar{\omega}^{a}\right\rangle_{J_{\chi, K}, K}=-h^{a}
$$

It follows from Eqn.(4.2)that

$$
\frac{\delta_{R} \Gamma[\chi, K]}{\delta K^{a}[\bar{\omega}]}=-h^{a},
$$

for the corresponding antisource $K^{a}[\bar{\omega}]$. Since this independent of $K^{A}$, it follows that $\Gamma[\chi, K]$ is linear in the antisource for $\bar{\omega}^{a}$. A similar argument holds for $\bar{\omega}_{\mu}^{a}, n^{a}$ and $\bar{\beta}^{a}$. Let me now look at the antisources for the remaining fields in the theory. The quantum effective action $\Gamma[\chi, K]$ must be linear in the antisources of $\theta^{a}$ and $C_{\mu}^{a}$, since these objects have mass dimension 3 and all other antisources have mass dimension 2. So $\Gamma[\chi, K]$ is at most quadratic 
in the antisources of only the other fields. It turns out that $\Gamma[\chi, K]$ is in fact linear in the remaining antisources as well. The argument involves showing that the coefficients of the quadratic terms are forced to vanish, term by term, by the dimensions and ghost numbers of the fields which can possibly appear in them. Appendix A contains the details of the argument.

It follows then that the effective action is at most linear in all the antisources $K^{A}$, and the arguments following Eqn.(4.7) hold. But the number of possible terms in the effective action allowed by the (renormalized) BRST symmetry $s_{R}$ is still enormous, and it is necessary to invoke other symmetries to simplify calculations.

Let me now consider the effect of the gauge-dependent symmetry $\tilde{s}$ on the effective action. I take the same partition function $Z[J, K]$ and the same effective action $\Gamma[\chi, K]$ as in Eqs. (4.1) and (4.2), with the same sources $J_{\chi, K}^{A}$ and the same antisources $K^{A}$. (This $\Gamma[\chi, K]$ was shown to be linear in these $K^{A}$ in Appendix A.) Let me also denote the minimal fields by $\phi^{A}$ and non-minimal fields by $\lambda^{A}$. Then $\tilde{s} \phi^{A}=s \phi^{A}=F^{A}$, and consequently $\tilde{s} F^{A}[\phi]=0$. The application of $\tilde{s}$ on the partition function gives (since the tree-level action $S$ is invariant under $\tilde{s})$,

$$
-\int d^{4} x\left[\left\langle F^{A}\right\rangle_{J_{\chi, K}, K} \frac{\delta_{L} \Gamma[\chi, K]}{\delta \phi^{A}}+\left\langle\tilde{s} \lambda^{A}\right\rangle_{J_{\chi, K}, K} \frac{\delta_{L} \Gamma[\chi, K]}{\delta \lambda^{A}}+\left\langle\tilde{s} s \lambda^{A}\right\rangle_{J_{\chi, K}, K} K^{A}[\lambda]\right]=0 .
$$

Now, if the gauge-fixing functions are linear in the fields, $\tilde{s} \lambda^{A}$ as defined in Eqn.(3.12) is either linear in the fields or equals the BRST variation of some linear function of the fields. Therefore, $\left\langle\tilde{s} \lambda^{A}\right\rangle_{J_{\chi, K}, K}$ is known in principle from solving the Zinn-Justin equations. In addition, the effective action does not contain the antisources corresponding to $\left(h^{a}, h_{\mu}^{a}, \alpha^{a}, \bar{\alpha}^{a}\right)$ and only $S_{R}$ contains the antisources for $\left(\bar{\omega}^{a}, \bar{\omega}_{\mu}^{a}, n^{a}, \bar{\beta}^{a}\right)$. Then I can read off from Eqn.(4.15) that $\Gamma_{N}^{(\epsilon)}[\chi]$ as defined in Eqn.(4.9) is invariant under $\tilde{s}_{R}$, which is just $\tilde{s}$ as calculated in terms of $s_{R}$. In other words, $\Gamma_{N}^{(\epsilon)}$ is invariant under $\tilde{s}_{R}$ where

$$
\begin{aligned}
\tilde{s}_{R} \bar{\omega}^{a} & =h^{a}+\frac{2}{\xi} f^{a}, \\
\tilde{s}_{R} h^{a} & =-\frac{2}{\xi} s_{R} f^{a} \equiv-\frac{2}{\xi} \Delta_{R}^{a}, \\
\tilde{s}_{R} \bar{\omega}_{\mu}^{a} & =h_{\mu}^{a}+\frac{2}{\eta} \partial_{\mu} n^{a}+\frac{2}{\eta} f_{\mu}^{a}, \\
\tilde{s}_{R} h_{\mu}^{a} & =\frac{2}{\eta}\left(\partial_{\mu} \alpha^{a}+\frac{2}{\zeta} \partial_{\mu} f^{\prime a}-s_{R} f_{\mu}^{a}\right) \equiv \frac{2}{\eta}\left(\partial_{\mu} \alpha^{a}+\frac{2}{\zeta} \partial_{\mu} f^{\prime a}-\Delta_{R \mu}^{a}\right), \\
\tilde{s}_{R} n^{a} & =-\left(\alpha^{a}+\frac{2}{\zeta} f^{\prime a}\right) \\
\tilde{s}_{R} \bar{\beta}^{a} & =-\left(\bar{\alpha}^{a}-\frac{2}{\zeta}, \partial_{\mu} \bar{\omega}^{a \mu}\right) \\
\tilde{s}_{R} \alpha^{a} & =-\frac{2}{\zeta} s_{R} f^{\prime a} \equiv-\frac{2}{\zeta} \Delta_{R}^{\prime a}, \\
\tilde{s}_{R} \bar{\alpha}^{a} & =\frac{2}{\zeta} \partial_{\mu}\left(h^{a \mu}+\frac{2}{\eta} \partial^{\mu} n^{a}+\frac{2}{\eta} f^{a \mu}\right), \\
\tilde{s}_{R} & =s_{R} \text { on all other fields. }
\end{aligned}
$$


Note that I did not fully utilize the nilpotence of $\tilde{s}$ itself. In principle, I could have treated $\tilde{s}$ just like $s$, defining new antisources $\widetilde{K}^{A}$ and deriving an analogue of Zinn-Justin equation. But that creates a host of other problems. In particular, the effective action is not linear in these new antisources $\widetilde{K}^{A}$.

These two renormalized symmetries, $s_{R}$ and $\tilde{s}_{R}$ are sufficient to uniquely fix the form of the effective action, as will be shown in the next section. There is a further symmetry which helps to pin down the form of $s_{R}$. This symmetry mixes the ghost fields with the same global properties and quantum numbers.

The action $S$ is invariant under

$$
\begin{aligned}
\delta \bar{\omega}^{a} & =-\delta \lambda \bar{\alpha}^{a}, \\
\delta \omega_{\mu}^{a} & =\delta \lambda\left(\partial_{\mu} \omega^{a}+g f^{a b c} A_{\mu}^{b} \omega^{c}\right), \\
\delta \theta^{a} & =-\delta \lambda \omega^{a}, \\
\delta \beta^{a} & =\delta \lambda \frac{1}{2} g f^{a b c} \omega^{b} \omega^{c}, \\
\delta(\text { all others }) & =0,
\end{aligned}
$$

where $\delta \lambda$ is a commuting c-number infinitesimal. It is straightforward to calculate that

$$
t s \theta^{a}=\frac{1}{2} g f^{a b c} \omega^{b} \omega^{c}, \quad t s(\text { all others })=0,
$$

where $t$ is the transformation $\delta / \delta \lambda$. Note that I have taken $\delta \lambda$ to be commuting only for convenience. If $\delta \lambda$ is taken to be anticommuting, the action will still be symmetric under $t=\delta_{L} / \delta \lambda$ provided $\delta_{L} \bar{\omega}^{a} / \delta \lambda=+\bar{\alpha}^{a}$, other transformation rules remaining the same. It is easy to see that the action $S$ is symmetric under $t$ for a large class of gauge-fixing functions $f^{a \mu}$.

By applying $t$ on the partition function (4.1), I get the Ward identities

$$
\int d^{4} x\left(\left\langle t \bar{\omega}^{a}\right\rangle \frac{\delta_{L} \Gamma}{\delta \bar{\omega}^{a}}+\left\langle t \omega_{\mu}^{a}\right\rangle \frac{\delta_{L} \Gamma}{\delta \omega_{\mu}^{a}}+\left\langle t \theta^{a}\right\rangle \frac{\delta_{L} \Gamma}{\delta \theta^{a}}+\left\langle t \beta^{a}\right\rangle \frac{\delta_{L} \Gamma}{\delta \beta^{a}}-\left\langle t s \theta^{a}\right\rangle K^{a}[\theta]\right)=0,
$$

where the quantum averages \langle\rangle are calculated in the presence of the currents and antisources $J_{\chi, K}, K$ as before, and $K^{a}[\theta]$ is the antisource for $\theta^{a}$. Since $t \theta^{a}=-\omega^{a}, t \bar{\omega}^{a}=-\bar{\alpha}^{a}$ are linear in the fields, their quantum averages are the same. As for the other two, $t \omega_{\mu}^{a}=s A_{\mu}^{a}$ and $t \beta^{a}=-s \omega^{a}$, so the quantum averages of the quantities on the left hand side are known. I can then write this equation as

$$
\int d^{4} x\left(-\bar{\alpha}^{a} \frac{\delta_{L} \Gamma}{\delta \bar{\omega}^{a}}+\frac{\delta_{R} \Gamma}{\delta K^{a \mu}[A]} \frac{\delta_{L} \Gamma}{\delta \omega_{\mu}^{a}}-\omega^{a} \frac{\delta_{L} \Gamma}{\delta \theta^{a}}-\frac{\delta_{R} \Gamma}{\delta K^{a}[\omega]} \frac{\delta_{L} \Gamma}{\delta \beta^{a}}+\frac{\delta_{R} \Gamma}{\delta K^{a}[\omega]} K^{a}[\theta]\right)=0 .
$$

Expanding $\Gamma$ in a power series in $\hbar$ and using arguments as before, I can write the divergent part of this equation as

$$
\begin{aligned}
\int d^{4} x\left(-\bar{\alpha}^{a} \frac{\delta_{L} \Gamma_{N, \infty}}{\delta \bar{\omega}^{a}}+\right. & \frac{\delta_{R} S_{R}}{\delta K^{a \mu}[A]} \frac{\delta_{L} \Gamma_{N, \infty}}{\delta \omega_{\mu}^{a}}+\frac{\delta_{R} \Gamma_{N, \infty}}{\delta K^{a \mu}[A]} \frac{\delta_{L} S_{R}}{\delta \omega_{\mu}^{a}}-\omega^{a} \frac{\delta_{L} \Gamma_{N, \infty}}{\delta \theta^{a}} \\
& \left.-\frac{\delta_{R} S_{R}}{\delta K^{a}[\omega]} \frac{\delta_{L} \Gamma_{N, \infty}}{\delta \beta^{a}}-\frac{\delta_{R} \Gamma_{N, \infty}}{\delta K^{a}[\omega]} \frac{\delta_{L} S_{R}}{\delta \beta^{a}}+\frac{\delta_{R} \Gamma_{N, \infty}}{\delta K^{a}[\omega]} K^{a}[\theta]\right)=0
\end{aligned}
$$


The $K$ independent terms of this equation give

$$
\int d^{4} x\left(-\bar{\alpha}^{a} \frac{\delta_{L} \Gamma^{(\epsilon)}[\chi]}{\delta \bar{\omega}^{a}}-F_{\mu}^{(\epsilon) a}[A] \frac{\delta_{L} \Gamma^{(\epsilon)}[\chi]}{\delta \omega_{\mu}^{a}}-\omega^{a} \frac{\delta_{L} \Gamma^{(\epsilon)}[\chi]}{\delta \theta^{a}}-F^{(\epsilon) a}[\omega] \frac{\delta_{L} \Gamma^{(\epsilon)}[\chi]}{\delta \beta^{a}}\right)=0,
$$

where $\Gamma^{(\epsilon)}[\chi]$ and $F^{(\epsilon)}[\chi]$ are as defined in Eqs. (4.9) and (4.11) with the indices $N$ and $x$ suppressed, and I have used the invariance of $S_{R}$ under the transformation $t$. The terms of first order in the antisources give

$$
\int d^{4} x\left(-\bar{\alpha}^{a} \frac{\delta_{L}}{\delta \bar{\omega}^{a}}-F_{\mu}^{(\epsilon) a}[A] \frac{\delta_{L}}{\delta \omega_{\mu}^{a}}-\omega^{a} \frac{\delta_{L}}{\delta \theta^{a}}-F^{(\epsilon) a}[\omega] \frac{\delta_{L}}{\delta \beta^{a}}\right) F^{(\epsilon) B}[\chi]=0
$$

for all $B$ except when $B$ corresponds to $\theta^{a}$, where I have used the fact that $t s=0$ on all fields except $\theta^{a}$. For the case of $K^{a}[\theta]$, this equation is modified,

$$
\int d^{4} x\left\{\left(-\bar{\alpha}^{b} \frac{\delta_{L}}{\delta \bar{\omega}^{b}}-F_{\mu}^{(\epsilon) b}[A] \frac{\delta_{L}}{\delta \omega_{\mu}^{b}}-\omega^{b} \frac{\delta_{L}}{\delta \theta^{b}}-F^{(\epsilon) b}[\omega] \frac{\delta_{L}}{\delta \beta^{b}}\right) F^{(\epsilon) a}[\theta]+F^{(\epsilon) a}[\omega]\right\}=0,
$$

upon using $t s \theta^{a}=\frac{1}{2} g f^{a b c} \omega^{b} \omega^{c} \equiv-F^{a}[\omega]$.

The interpretation of these equations is obvious. Eqn.(4.22) says that $\Gamma_{N}^{(\epsilon)}[\chi]$ is invariant under $t_{R}$, where

$$
\begin{aligned}
t_{R} \bar{\omega}^{a} & =-\bar{\alpha}^{a}, \\
t_{R} \omega_{\mu}^{a} & =s_{R} A_{\mu}^{a}, \\
t_{R} \theta^{a} & =-\omega^{a}, \\
t_{R} \beta^{a} & =-s_{R} \omega^{a}, \\
t_{R}(\text { all others }) & =0,
\end{aligned}
$$

The first equation following that, Eqn.(4.23), shows that $t_{R} s_{R}=0$ on all fields except $\theta^{a}$, and Eqn.(4.24) shows that $t_{R} s_{R} \theta^{a}=-s_{R} \omega^{a}$. There are no surprises, except perhaps the fact that these conditions are actually useful in restricting the form of $s_{R}$ to what is shown in Appendix B.

\section{THE MOST GENERAL EFFECTIVE ACTION}

In this section I shall use brute force methods to show that the effective action contains the same terms, up to arbitrary multiplicative renormalizations, as the tree-level action of Eqn.(3.2). The proof requires construction of the most general nilpotent transformation of the fields, $s_{R}$, as discussed in the previous section. The actual construction of $s_{R}$ is a rather involved digression, so I have separated it into Appendix B. The result of $s_{R}$ on the various fields is given in Eqn.(B32).

Now I need to construct the most general functional $\Gamma_{N}^{(\epsilon)}$ symmetric under $s_{R}$ as well as under the linear symmetries of $S$. These are $(i)$ Lorentz invariance, $(i i)$ global $S U(N)$ invariance, and (iii) ghost number conservation. However, it is obvious that even with the restrictions imposed by $s_{R}$ and these three symmetries, there is an enormous number 
of possible terms. Since $C_{\mu}^{a}$ has mass dimension zero as well as ghost number zero, it is possible to multiply any other term by a scalar polynomial of the form $\sum_{k=1}^{n}\left(C_{\mu}^{a} C^{a \mu}\right)$, and still maintain the three abovementioned symmetries. Of course, such terms are not $s_{R}$-invariant by themselves, but one can imagine that their variations cancel against those of something else, and ruling out each such term requires a long and tedious calculation. And even without this unwanted complexity, there are of the order of one hundred terms satisfying the three linear symmetries. Applying $s_{R}$ to a sum of so many terms, multiplied by unknown scalar polynomials of $C_{\mu}^{a}$, and then finding the combination which remains invariant, would require unlimited time and perseverance. Fortunately, there is a way out of this quagmire, provided by $\tilde{s}$.

As before, let me denote the minimal fields by $\phi^{A}$ and non-minimal fields by $\lambda^{A}$. Let me also define $s_{R}^{\prime} \equiv \frac{1}{2}\left(s_{R}-\tilde{s}_{R}\right)$. Then from Eqn.(4.15),

$$
s_{R}^{\prime} \phi^{A}=0, s_{R}^{\prime} \lambda^{A} \neq 0 \text {. }
$$

The non-minimal fields $\lambda^{A}$ are $\left(\bar{\omega}^{a}, \bar{\beta}^{a}, \bar{\omega}_{\mu}^{a}, \bar{\alpha}^{a}, h^{a}, \alpha^{a}, h_{\mu}^{a}, n^{a}\right)$. Let me also choose the gauge fixing functions to be specifically those in Eqn. (3.1). Because of my choice of gauge-fixing functions, the action exhibits invariance under constant shifts of $\bar{\omega}^{a}, \bar{\beta}^{a}, \bar{\omega}_{\mu}^{a}$ and $n^{a}$. Since these are linear symmetries, I can impose them on the effective action. In other words, these fields must appear in the quantum effective action only as derivatives, i.e., as $\partial_{\mu} \bar{\omega}^{a}$ etc.

Then on dimensional grounds, the effective action will be at most quadratic in the $\lambda^{A}$. I can then write the effective action in the generic form

$$
\Gamma=\sum_{A} \lambda^{A} X^{A}+\sum_{A, B} \lambda^{A} \lambda^{B} X^{A B}
$$

where $X^{A}$ and $X^{A B}$ do not contain any of the $\lambda^{A}$, and have appropriate transformation properties, dimension and ghost number. In particular, $X^{A}$ and $X^{A B}$ are assumed to include derivative operators as necessary for the constant shift symmetries mentioned above, and the sum over indices will be taken to include an integral over space-time unless specified otherwise. Since both $s_{R}$ and $\tilde{s}_{R}$ are symmetries of the effective action, I have

$$
s_{R}^{\prime} \Gamma=0
$$

and from Eqn.(5.1), I have

$$
s_{R}^{\prime} X^{A}=s_{R}^{\prime} X^{A B}=0
$$

Therefore using Eqn.(5.2) I can write

$$
\sum_{A}\left(s_{R}^{\prime} \lambda^{A}\right) X^{A}+\sum_{A, B}\left(s_{R}^{\prime} \lambda^{A}\right) \lambda^{B} X^{A B}+\sum_{A, B}(-1)^{\varepsilon_{A}} \lambda^{A}\left(s_{R}^{\prime} \lambda^{B}\right) X^{A B}=0 .
$$

Here $\varepsilon_{A}$ (not to be confused with the $\varepsilon_{A}$ of Eqs. (A3) and (A4)) is the Grassmann parity of the field $\lambda^{A}$.

Since $X^{A}$ and $X^{A B}$ do not contain any of the $\lambda^{A}$ by definition, I can now look at the coefficients of the various $\lambda^{A}$ in the expansion of Eqn.(5.5) and set them to zero in order 
to get an expression for the effective action $\Gamma$. Many of the terms are thus eliminated, and some algebraic relations appear among some of the rest. The details of the calculation are given in Appendix Q. The result is the effective Lagrangian of Eqn.(C41) which I give here again,

$$
\begin{aligned}
\mathscr{L}_{g}=\bar{\omega}^{a} X_{\bar{\omega}}^{a} & +\bar{\beta}^{a} X_{\bar{\beta}}^{a}+\bar{\omega}_{\mu}^{a} X_{\bar{\omega}_{*}}^{a \mu}+\bar{\alpha}^{a} X_{\bar{\alpha}}^{a}+h^{a} X_{h}^{a}+h_{\mu}^{a} X_{h_{*}}^{a \mu}+\partial_{\mu} n^{a} X_{n}^{a \mu} \\
& +\bar{\omega}_{\mu}^{a} \alpha^{b} X_{\bar{\omega}_{*} \alpha}^{a b \mu}+\bar{\alpha}^{a} \alpha^{b} X_{\bar{\alpha} \alpha}^{a b}+h^{a} h^{b} X_{h h}^{a b}+h^{a}\left(\eta h_{\mu}^{b}+\partial_{\mu} n^{b}\right) X_{h n}^{a b \mu} \\
& +h_{\mu}^{a} h_{\nu}^{b} X_{h_{*} h_{*}}^{a b \mu \nu}+h_{\mu}^{a} \partial_{\nu} n^{b} X_{h_{*} n}^{a b \mu \nu}+\partial_{\mu} n^{a} \partial_{\nu} n^{b} X_{n n}^{a b \mu \nu} .
\end{aligned}
$$

The undetermined coefficients satisfy several relations among themselves as shown in Appendix 9 .

The number of unknown coefficients can be reduced even further. Just as the symmetry $s_{R}^{\prime} \Gamma=0$ produced relations among several of these $X$ 's, the quantum BRST symmetry itself, $s_{R} \Gamma=0$, should produce some more relations independent of the previous ones. The expression for the $s_{R}$-variation is

$$
\begin{aligned}
s_{R} \Gamma= & \sum_{A}\left[\left(s_{R} \lambda^{A}\right) X^{A}+(-1)^{\varepsilon_{A}} \lambda^{A}\left(s_{R} X^{A}\right)\right] \\
& +\sum_{A B}\left[\left(s_{R} \lambda^{A}\right) \lambda^{B} X^{A B}+(-1)^{\varepsilon_{A}} \lambda^{A}\left(s_{R} \lambda^{B}\right) X^{A B}+(-1)^{\varepsilon_{A}+\varepsilon_{B}} \lambda^{A} \lambda^{B}\left(s_{R} X^{A B}\right)\right]=0 .
\end{aligned}
$$

Since $s_{R} X^{A}$ and $s_{R} X^{A B}$ do not contain any of the $\lambda^{A}$, I can consider the coefficients of $\lambda^{A}$ or of $\lambda^{A} \lambda^{B}$ in the above expression and set them to zero.

The calculation is fairly straightforward, but in keeping with other calculations in this paper, I have again separated this one into Appendix D. The result is that the functional forms of all the unknown coefficients become known, and only two arbitrary constants are needed to write them, as shown in Eqn. (D19).

I can now write down the general form of the ghost sector of the theory as

$$
\begin{gathered}
\mathscr{L}_{g}=Z_{\omega} \bar{\omega}^{a} \Delta_{R}^{a}+Z_{\beta} \bar{\beta}^{a} \Delta_{R}^{\prime a}+Z_{\beta} \bar{\omega}_{\mu}^{a} \Delta_{R}^{a \mu}+Z_{\beta} \bar{\alpha}^{a} f^{\prime a}+Z_{\omega} h^{a} f^{a}+Z_{\beta} h_{\mu}^{a}\left(f^{a \mu}+\partial^{\mu} n^{a}\right) \\
-Z_{\beta} \partial^{\mu} \bar{\omega}_{\mu}^{a} \alpha^{a}+\zeta Z_{\beta} \bar{\alpha}^{a} \alpha^{a}+\frac{\xi}{2} Z_{\omega} h^{a} h^{a}+\frac{\eta}{2} Z_{\beta} h_{\mu}^{a} h^{a \mu} .
\end{gathered}
$$

It remains to construct the most general non-ghost sector of the theory.

The BRST transformations on the bosonic fields as found in Eqn.(B32) of the Appendix are given by

$$
\begin{aligned}
s_{R} A_{\mu}^{a} & =\partial_{\mu} \omega_{R}^{a}+g_{R} f^{a b c} A_{\mu}^{b} \omega_{R}^{c}, \\
s_{R} B_{\mu \nu}^{a} & \left.=g_{R} f^{a b c} B_{\mu \nu}^{b} \omega_{R}^{c}+\partial_{[\mu} \omega_{R \nu]}^{a}+g_{R} f^{a b c} A_{[\mu}^{b} \omega_{R \nu]}^{c}+g_{R} f^{a b c} \partial_{[\mu} A_{\nu]}^{b} \theta_{R}^{c}+g_{R}^{2} f^{a e d} f^{e b c} A_{\mu}^{b} A_{\nu}^{c} \theta_{R}^{d}\right), \\
s_{R} C_{\mu}^{a} & =g_{R} f^{a b c} C_{\mu}^{b} \omega_{R}^{c}+\frac{\mathscr{N}_{4}}{\mathscr{N}_{6}}\left(\omega_{R \mu}^{a}+\partial_{\mu} \theta_{R}^{a}+g_{R} f^{a b c} A_{\mu}^{b} \theta_{R}^{c}\right),
\end{aligned}
$$

where I have defined $g_{R}=\frac{g}{\mathscr{N}_{1}}, \omega_{R}^{a}=\mathscr{Z} \mathscr{N}_{1} \omega^{a}, \omega_{R \mu}^{a}=\mathscr{Z} \mathscr{N}_{6} \omega_{\mu}^{a}$ and $\theta_{R}^{a}=\mathscr{Z} \mathscr{N}_{6} \mathscr{N}_{1} \theta^{a}$. If I now define renormalized field strengths 


$$
\begin{aligned}
\tilde{F}_{\mu \nu}^{a} & =\partial_{\mu} A_{\nu}^{a}-\partial_{\nu} A_{\mu}^{a}+g_{R} f^{a b c} A_{\mu}^{b} A_{\nu}^{c} \\
\tilde{H}_{\mu \nu \lambda}^{a} & =\partial_{[\mu} B_{\nu \lambda]}^{a}+g_{R} f^{a b c} A_{[\mu}^{b} B_{\nu \lambda]}^{c}-\frac{\mathscr{N}_{6}}{\mathscr{N}_{4}} g_{R} f^{a b c} \tilde{F}_{[\mu \nu}^{b} C_{\lambda]}^{c},
\end{aligned}
$$

I find that the Zinn-Justin equation just says that the ghost-free sector is invariant under these gauge transformations. The factor $\mathscr{N}_{6} / \mathscr{N}_{4}$ can be absorbed either in $C_{\mu}^{a}$ itself or in the renormalization of a fiduciary coupling constant $g_{C}$ which always appears in front of $C_{\mu}^{a}$.

The procedure described so far can be used to construct effective actions for different theories involving the non-Abelian two-form. For example, it may be interesting to apply it to the recently proposed first-order formulation of Yang-Mills theory [16. However, since I have a specific theory in mind, I will need to invoke another symmetry in order to eliminate unwanted terms from the non-ghost sector.

This 'symmetry' was an invariance of the classical equations of motion under

$$
B_{\mu \nu}^{a} \rightarrow B_{\mu \nu}^{a}+\alpha F_{\mu \nu}^{a}
$$

with $\alpha$ a constant. It was suggested in [8] that this symmetry could play a role in preventing terms of the form $(B-D C)^{2}$ or $(B-D C) \wedge(B-D C)$ from appearing in the action. Of course, since the classical action is not invariant but changes by a total derivative, it is nontrivial to elevate this to a quantum symmetry. Classically this 'symmetry' leads to a conserved current

$$
J_{T}^{\mu}=H^{a \mu \nu \lambda} F_{\nu \lambda}^{a}+m \epsilon^{\mu \nu \lambda \rho}\left(A_{\nu}^{a} F_{\lambda \rho}^{a}-\frac{2}{3} f^{a b c} A_{\nu}^{a} A_{\lambda}^{b} A_{\rho}^{c}\right) .
$$

But there is no corresponding conserved current for the BRST-invariant action $S$ of Eqn.(3.2), which was the starting point of quantization. This part of the problem can be circumvented quite easily by incorporating the shift into the BRST transformation for $B_{\mu \nu}^{a}$, which now reads (cf. Eqn.(3.3))

$$
s B_{\mu \nu}^{a}=g f^{a b c} B_{\mu \nu}^{b} \omega^{c}+\partial_{[\mu} \omega_{\nu]}^{a}+g f^{a b c} A_{[\mu}^{b} \omega_{\nu]}^{c}+g f^{a b c} F_{\mu \nu}^{b} \theta^{c}+\alpha F_{\mu \nu}^{a} .
$$

Here $\alpha$ is an anticommuting constant with ghost number +1 , and $s \alpha=0$. It is trivial to see that the BRST transformation $s$ is still nilpotent, $s^{2}=0$. However, the action is still not invariant, but is shifted by $\frac{m \alpha}{4} \int d^{4} x \epsilon^{\mu \nu \lambda \rho} F_{\mu \nu}^{a} F_{\lambda \rho}^{a}$. So it seems that I have not gained anything, but only recovered a symmetry of the equations of motion. On the other hand, since I am now dealing with the quantum theory rather than the classical action principle, I can also generate this term through quantum effects.

For example, this term could be canceled by the transformation of the fermion measure if fermions are coupled to the gauge field. Under a chiral transformation with a parameter $4 \pi^{2} m \alpha$, the effective action changes by $-\frac{m \alpha}{4} \int d^{4} x \epsilon^{\mu \nu \lambda \rho} F_{\mu \nu}^{a} F_{\lambda \rho}^{a}$, which cancels the effect of the shift transformation of Eqn. (5.11). The action then becomes invariant under the combination of the shift and global chiral transformations. There are other ways of canceling the term generated by the shift. In any case, symmetry under the shift transformation rules out terms involving products of $\left(B_{\mu \nu}-D_{[\mu} C_{\nu]}\right)$. I can then write down the most general quantum effective action consistent with the quantum symmetries,

$$
\Gamma_{\text {eff }}=\int d^{4} x\left(Z_{A} \tilde{F}_{\mu \nu}^{a} \tilde{F}^{a \mu \nu}+Z_{B} \tilde{H}_{\mu \nu \lambda}^{a} \tilde{H}^{a \mu \nu \lambda}+Z_{B F} m \epsilon^{\mu \nu \lambda \rho} B_{\mu \nu}^{a} \tilde{F}_{\lambda \rho}^{a}+\mathscr{L}_{g}\right) .
$$

This is the same as the tree-level action up to arbitrary multiplicative constants, which means that the theory is perturbatively renormalizable. 


\section{DISCUSSION OF RESULTS}

It is time to gather the results. I have given an algebraic proof of perturbative renormalizability of the dynamical non-Abelian two-form gauge theory, also known as the topological mass generation mechanism. It follows that just as in two and three dimensions, it is possible in four dimensions to have a renormalizable theory of massive non-Abelian vector bosons without a residual Higgs particle.

The calculations were done in a specific set of linear gauges, so that antighosts appeared only as derivatives. In other linear gauges, the calculations would be more involved, in particular there would be terms cubic and quartic in the antighosts, but even in such cases the methods of Sec. $\mathrm{V}$ should go through. Two other symmetries appeared as a result of using linear gauges - these are $\tilde{s}$, defined in Eqn. (3.12), and $t$, defined in Eqn. (4.17). These two symmetries were greatly useful for constructing the quantum BRST symmetry and for reducing the number of possible terms in the quantum effective action. These symmetries would be present in other linear gauges as well, but not in a general nonlinear gauge. The calculations are extremely tedious for nonlinear gauges, and it is not clear if the quantum BRST symmetry alone is sufficient to restrict the terms in the quantum effective action to the same form as those in the tree-level action in such gauges.

The proof also depends crucially on the nature of the auxiliary field $C_{\mu}^{a}$. There is no quadratic term for this field in the action of Eqn. (2.2), and it was mentioned in Sec. II that as a result there was no propagator for $C_{\mu}^{a}$ and diagrams with internal $C_{\mu}^{a}$ lines vanished. This seems rather peculiar, but it is actually not a problem for perturbation theory as long as there are other fields which propagate freely. The free Hamiltonian can always be written in terms of the propagating free fields, and other terms can be thought of as perturbation on top of it, with $C_{\mu}^{a}$ being a non-dynamical field. One may question whether perturbation theory is valid for an action for which the operator in the matrix of quadratic terms is not invertible, as in this case. In general, perturbation can be done only if a free Hamiltonian can be constructed for the theory. I have assumed that this is a sufficient condition as well, as long as all the physical fields appear in the free Hamiltonian and their quadratic matrix can be inverted. The free Hamiltonian is a sum of terms like $\frac{1}{2}\left(\Pi^{2}+\Phi^{2}\right)$ only over the physical degrees of freedom, and this is the part which gives the propagators. So if the number of degrees of freedom in the theory is known, it is strictly necessary to have only that many propagators, and therefore only that many quadratic pieces in the theory. The quadratic matrix of the physical fields in this theory can be inverted, as can be seen from the fact that perturbatively there are three propagating degrees of freedom, as there should be from counting constraints. In other words, the assumption made here is that for a perturbation series to be constructed, only physical degrees of freedom need to be identified with quantum fields. The non-propagating degrees need not be quantum fields in the sense of canonical quantization as long as the path integral can be formally constructed for them. This is not a radical assumption, it is made for all gauge theories, but is usually associated with unphysical objects (such as the scalar mode of a vector field) which are not Lorentz covariant. On the other hand, it is clear that $C_{\mu}^{a}$ by itself is not a physical field, as it can be completely removed by a vector gauge transformation.

Therefore I can try to choose a gauge in which the path integral over $C_{\mu}^{a}$ can be formally

calculated in the Lagrangian formalism and in which there is a propagator for $C_{\mu}^{a}$. The 
gauge chosen in Sec. [II] was $f_{\mu}^{a}=\partial^{\nu} B_{\mu \nu}^{a}$ which did not give a propagator for $C_{\mu}^{a}$. For convenience, let me keep to linear gauges so that the proof given above can be used with minimal modifications. Since $C_{\mu}^{a}$ can be shifted away, it would seem that the gauge $C_{\mu}^{a}=0$, i.e. the choice of $f_{\mu}^{a}=m^{2} C_{\mu}^{a}$ could provide the necessary term. However, this is not a good gauge choice. The vector gauge transformations get fixed completely, but now there is no propagator for $B_{\mu \nu}^{a}$ as the corresponding quadratic operator is non-invertible. One possible alternative is to choose an $R_{\xi}$ type gauge, $f_{\mu}^{a}=\partial^{\nu} B_{\mu \nu}^{a}+\eta m^{2} C_{\mu}^{a}$. In this gauge there is no canonical momentum for $C_{\mu}^{a}$ (which was true for the classical theory also), so $C_{\mu}^{a}$ terms do not appear in the free Hamiltonian (unlike the apparently analogous case of a Goldstone mode in broken gauge theories). But formally the Lagrangian path integral over $C_{\mu}^{a}$ can be done, and formally $C_{\mu}^{a}$ will have a propagator as well as a two-point vertex with $B_{\mu \nu}^{a}$ after using the equations of motion for $h_{\mu}^{a}$ and $n^{a}$. The total tree-level propagator will be a sum over insertions of these vertices as well as vertices between $A_{\mu}^{a}$ and $B_{\mu \nu}^{a}$. This propagator will be gauge-dependent,

$$
\widetilde{\Delta}_{C \mu \nu}^{a b}=-\frac{\delta^{a b}}{m^{4}} \frac{k^{2}-m^{2}}{(\eta-1) k^{2}-\eta m^{2}} .
$$

This propagator clearly does not represent a real particle because it has a gauge dependent pole, as is expected because of the Goldstone-like nature of $C_{\mu}^{a}$. This propagator also has the ultraviolet behavior of $\Delta_{C \mu \nu}^{a b} \sim O(1)$ as $k^{\mu} \rightarrow \infty$, which is not very good for proofs of renormalizability, although because of the gauge-dependence of the pole in $\Delta_{C \mu \nu}^{a b}$ it may not be relevant. However, in this gauge the action is not invariant under a constant shift of $\bar{\omega}_{\mu}^{a}$, so the proof given above will not be applicable directly but will have to be redone after including cubic and quadratic terms in $\bar{\omega}_{\mu}^{a}$.

Another possibility for an $R_{\xi}$ type gauge choice is $f_{\mu}^{a}=\partial^{\nu} B_{\mu \nu}^{a}+\eta \square C_{\mu}^{a}$. The gauge-fixed theory in this gauge also does not have a canonical momentum for $C_{\mu}^{a}$ and therefore terms involving $C_{\mu}^{a}$ do not appear in the free Hamiltonian. But again the Lagrangian path integral over $C_{\mu}^{a}$ can be done formally, and after summing over all two-point vertex insertions, the total tree-level propagator will again be gauge-dependent,

$$
\Delta_{C \mu \nu}^{a b}=-\frac{\delta^{a b}}{k^{4}} \frac{k^{2}-m^{2}}{(\eta-1) k^{2}-\eta m^{2}} .
$$

This (formal) propagator also has a gauge dependent pole and therefore cannot represent a real particle. On the other hand, $\Delta_{C \mu \nu}^{a b}$ falls off as $O\left(k^{-4}\right)$ in the ultraviolet regime. Since $C_{\mu}^{a}$ has been assumed to have a vanishing mass dimension, $\Delta_{C \mu \nu}^{a b}$ therefore satisfies the criterion for power-counting renormalizability that given a field of mass dimension $d$, its propagator should fall off as $O\left(k^{-\delta}\right)$, where $4-\delta \leq 2 d$ [14]. Therefore it can be safely used in algebraic proofs of renormalizability. And because of this particular choice of gauge, the antighost $\bar{\omega}_{\mu}^{a}$ still appears only with derivatives, and the action is still symmetric under a constant shift of $\bar{\omega}_{\mu}^{a}$, and the effective action is still at most quadratic in $\bar{\omega}_{\mu}^{a}$. Because this is a linear gauge, the rest of the arguments of the paper go through without modification, and we recover the same proof in this $R_{\xi}$-type gauge. In both these gauges, the propagators for the ghosts of the vector symmetry also have gauge-dependent poles.

Although the Lagrangian path integral over $C_{\mu}^{a}$ can be formally done in the above gauges, the nature and the role of the auxiliary field remain obscure. Even then this is somewhat 
better than a canonical analysis of the system, where it is not possible to identify $C_{\mu}^{a}$ with a quantum field because its canonical momentum vanishes. That quantization is problematic even in the Lagrangian path integral formalism is brought out by the fact that the calculations above remain the same even in the absence of a propagator for $C_{\mu}^{a}$. However, the essential point of this paper is the following - despite apparent problems with perturbative expansion of this theory, application of standard algebraic techniques to it leads to a quantum action which contains the same operators as the classical action, which would be a proof of renormalizability in any other theory. This result was completely unexpected on the grounds of the problems with perturbative expansion, already mentioned above and in [7].

The use of the anticommuting constant $\alpha$ can potentially create problems because it has vanishing mass dimension, but ghost number +1 , by Eqn. (5.13). The fact that there is an anticommuting constant in the theory is not in itself a problem, similar objects appear in supersymmetric quantum mechanics [17]. But the fact that it has vanishing mass dimension can cause problems of its own. One place where problems can arise is the argument in Sec. IV and particularly in Appendix A that the quantum effective action is at most linear in the antisources $K^{A}$. The argument relied on the fact that the coefficients of these quadratic terms had vanishing mass dimension but non-vanishing ghost number, so they must contain $\theta^{a}$. Now $\theta^{a}$ can be replaced by the constant $\alpha$. But there is no reason to worry, because the relevant objects have ghost number +2 or more, so at least one $\theta^{a}$ will be needed to construct any of them, and the rest of the argument remains unchanged. Another possible place for a problem is in the calculation of the general nilpotent transformation $s_{R}$, given in the Appendix B. Some of the fields $\chi^{A}$ could have a term like $\alpha \chi^{A}$ in their transformation rules in principle. Other similarly constructed terms are also possible. An explicit calculation using the $t$ symmetry shows that such terms do not arise.

I have not touched on the issue of anomalies, or the inclusion of fermions, in detail. Fermions will couple to the Yang-Mills gauge field in the usual way, but there is no gaugeinvariant coupling of mass dimension four between the two-form and fermions because of the shift symmetry mentioned in Eqn. (5.11). The Yang-Mills theory will have the usual SU(N) anomaly of $\operatorname{Tr} F \wedge F$. This can be removed by use of the shift symmetry. Gauge anomalies will be absent if the gauge group is the Standard Model gauge group. The two-form brings with it a vector gauge symmetry, as given in Eqn. (2.6). This is an Abelian symmetry, but there is no field carrying the charge corresponding to it. So there is no anomaly involving this transformation.

The original motivation for the theory was to find a possible alternative for the Higgs sector of the Salam-Weinberg model of electroweak interactions. What I have shown in this paper is that it is possible to have massive vector bosons without spontaneous symmetry breaking. But the problem with applying this mechanism to electroweak interactions is precisely that there is no symmetry breaking, whereas the observed world has broken $\mathrm{SU}(2) \times \mathrm{U}(1)$ symmetry. If I write the Lagrangian for the Salam-Weinberg model without the Higgs field, and add an SU(2) two-form with an action as in Eqn.(2.2), all the SU(2) gauge bosons get the same mass, contrary to experiment. All other observed events would remain uncontradicted. It has been suggested [11 that by also adding a U(1) two-form, which would make the photon massive, it may be possible to get the correct mass ratio of $\mathrm{Z}$ and $\mathrm{W}^{ \pm}$particles. However, agreement with experiment requires an infinite parameter in the classical Lagrangian, corresponding to an infinite mass for the photon. Another way is 
to add an explicit symmetry breaking term to the Lagrangian, so that the mass term reads

$$
\frac{m}{4} \epsilon^{\mu \nu \rho \lambda}\left(B_{\mu \nu}^{a} F_{\rho \lambda}^{a}-\tan \theta_{W} B_{\mu \nu}^{3} F_{\rho \lambda}\right),
$$

where $F_{\rho \lambda}$ is the field strength of the $\mathrm{U}(1)$ gauge field, and $\theta_{W}$ is the Weinberg angle. Then by defining the $\mathrm{Z}$ and photon fields as usual, I can write the quadratic part of this mass term as

$$
\frac{\epsilon^{\mu \nu \rho \lambda}}{4}\left(m B_{\mu \nu}^{1} \partial_{[\rho} A_{\lambda]}^{1}+m B_{\mu \nu}^{2} \partial_{[\rho} A_{\lambda]}^{2}+m \sec \theta_{W} B_{\mu \nu}^{3} \partial_{[\rho} Z_{\lambda]}\right)
$$

It follows from this that the $\mathrm{Z}$ is heavier than the $\mathrm{W}^{ \pm}$by a factor of $\sec \theta_{W}$ (and the photon is massless). However, because of the explicit symmetry breaking term, the proof of renormalizability given here is not applicable. So the question of applicability of this model to electroweak interactions remains open.

Acknowledgement: It is a pleasure to acknowledge long discussions with F. Barbero and E. Sanchez about the nature of the auxiliary vector field. 


\section{APPENDIX A: ANTISOURCE DEPENDENCE OF $\Gamma_{N, \infty}$}

As was argued in Sec. IV, the quantum effective action is at most quadratic in the antisources. In fact, several of the quadratic terms were eliminated just by looking at the mass dimensions and BRST transformation properties of the fields. In order to see the dependence of the effective action on the rest of the antisources, let me write the general expression of $\Gamma_{N, \infty}[\chi, K]$ as

$$
\Gamma_{N, \infty}[\chi, K]=\Gamma_{N, \infty}[\chi, 0]+\int d^{4} x \mathscr{F}_{N}^{A}[\chi, x] K^{A}(x)+\int d^{4} x \mathscr{F}_{N}^{A B}[\chi, x] K^{A}(x) K^{B}(x) .
$$

In this there is no $K^{A}$ corresponding to $h^{a}, h_{\mu}^{a}, \alpha^{a}$ and $\bar{\alpha}^{a}$, and the quadratic sum also does not run over the antisources for $\theta^{a}, C_{\mu}^{a}, \bar{\omega}^{a}, \bar{\omega}_{\mu}^{a}, n^{a}$ and $\bar{\beta}^{a}$ for reasons described in Sec. IV. The relation $\left(S_{R}, \Gamma_{N, \infty}\right)=0$, when applied to this expression, gives at zeroth order in $K^{A}$

$$
-\int d^{4} x \mathscr{F}_{N}^{A} \frac{\delta_{L} S_{R}[\chi, 0]}{\delta \chi^{A}}-\int d^{4} x F^{A} \frac{\delta_{L} \Gamma_{N, \infty}[\chi, 0]}{\delta \chi^{A}}=0
$$

At first order in $K^{A}$, I get the equation

$$
\int d^{4} x\left[\mathscr{F}_{N}^{B} \frac{\delta_{L} F^{A}}{\delta \chi^{B}}+F^{B} \frac{\delta_{L} \mathscr{F}_{N}^{A}}{\delta \chi^{B}}+2 \frac{\delta_{L} S_{R}[\chi, 0]}{\delta \chi^{B}} \mathscr{F}_{N}^{A B}\right]=0 .
$$

Here I have used the fact that $\mathscr{F}_{N}^{B A}=(-1)^{\varepsilon_{A} \varepsilon_{B}} \mathscr{F}_{N}^{A B}$ where $\varepsilon_{A}, \varepsilon_{B}$ are the Grassmann parities of $K^{A}$ and $K^{B}, 0$ for bosonic $K^{A}$ and 1 for fermionic $K^{A}$. The terms of second order in the antisources lead to the equation

$$
\begin{aligned}
\sum_{C} \int d^{4} x F^{C}(x) \frac{\delta_{L} \mathscr{F}_{N}^{A B}(y)}{\delta \chi^{C}(x)} \delta^{4}(y-z) & \\
& +\sum_{C}\left[(-1)^{\varepsilon_{A}\left(\varepsilon_{B}+\varepsilon_{C}+1\right)} \mathscr{F}_{N}^{A C}(y) \frac{\delta_{L} F^{B}(z)}{\delta \chi^{C}(y)}+A \leftrightarrow B\right]=0
\end{aligned}
$$

The coefficient $\mathscr{F}_{N}^{A B}[\chi, x]$ has mass dimension $d_{A}+d_{B}-2$, and ghost number $\gamma_{A}+\gamma_{B}+2$, where $d_{A}$ and $\gamma_{A}$ are respectively the mass dimension and ghost number of the field $\chi^{A}$. Since $\theta^{a}$ has ghost number +1 and mass dimension zero, it is possible to construct functions of arbitrary positive ghost number and mass dimension zero by taking products of $\theta^{a}$. Since the quadratic sum runs only over the antisources for the fields $A_{\mu}^{a}, B_{\mu \nu}^{a}, \omega^{a}, \omega_{\mu}^{a}, \beta^{a}$, it follows that $\mathscr{F}_{N}^{A B}$ can depend only on $\theta^{a}$ and $C_{\mu}^{a}$ for all $A, B$, and $\theta^{a}$ must be present in $\mathscr{F}_{N}^{A B}$ to take care of its ghost number, which is always positive. So the first term of Eqn. (A4), $F^{C} \delta_{L} \mathscr{F}_{N}^{A B} / \delta \chi^{C}$, must contain for all $A, B$,

$$
\left(s \theta^{a}\right) \frac{\delta_{L} \mathscr{F}_{N}^{A B}}{\delta \theta^{a}}=\left(-g f^{a b c} \theta^{b} \omega^{c}-\beta^{a}\right) \frac{\delta_{L} \mathscr{F}_{N}^{A B}}{\delta \theta^{a}} .
$$

The first term on the right hand side will always appear in $\mathscr{F}^{A C} \delta_{L} F^{B} / \delta \chi^{C}$ because $\mathscr{F}^{A C}$ contains $\theta^{a}$ for all $A, B$, but the second will appear only if $F^{A}$ contains $\beta^{a}$. (The index $N$ is suppressed from now on.) 
In the sum $\mathscr{F}^{A C} \delta_{L} F^{B} / \delta \chi^{C}$, the only terms that contribute a $\beta^{a}$ are for $\chi^{C}$ corresponding to $A_{\mu}^{a}$ when $\chi^{B}$ is $\omega_{\mu}^{a}$, and $\chi^{C}$ corresponding to $\omega^{a}$ when $\chi^{B}$ is $\beta^{a}$. This implies, first of all, that at least one of the indices $A, B$ in $\mathscr{F}^{A B}$ must correspond to either $\omega_{\mu}^{a}$ or $\beta^{a}$. In other words, when neither $\chi^{A}, \chi^{B}$ corresponds to $\omega_{\mu}^{a}$ or $\beta^{a}$, the sum $\mathscr{F}^{A C} \delta_{L} F^{B} / \delta \chi^{C}$ does not contain $\beta^{a}$ even after (anti-)symmetrization over $A, B$, while the sum $F^{C} \delta_{L} \mathscr{F}^{A B} / \delta \chi^{C}$ must contain $\beta^{a}$. Therefore $\mathscr{F}^{A B}=0$ for all such pairs $A, B$. So for example $\mathscr{F}^{a b}(\omega, \omega)=0$.

Now, the only $\mathscr{F}^{A C}$ which contribute a $\beta^{a}$ to the sum $\mathscr{F}^{A C} \delta_{L} F^{B} / \delta \chi^{C}$ are those for which one index corresponds to one of $\left(\omega_{\mu}^{a}, \beta^{a}\right)$, and the other index to $A_{\mu}^{a}$ or $\omega^{a}$ and all these $\mathscr{F}^{A C}$ contain only products of $\theta^{a}$ and $C_{\mu}^{a}$. Looking at $\mathscr{F}^{A B}$ of this type, I find that each term which can contribute a $\beta^{a}$ to the sum has a factor $\mathscr{F}^{A C}$ of the type that vanishes by the previous argument. For example, if $(A, B)$ correspond to $\left(\omega^{a}, \beta^{a}\right)$, the only term in the sum that could contribute a factor of $\beta^{a}$ is $\mathscr{F}^{a c}(\omega, \omega) \delta_{L} F^{b}(\beta) / \delta \omega^{c}$, which vanishes since $\mathscr{F}^{a c}(\omega, \omega)=0$. Explicitly, for this case Eqn. (A4) reads

$$
\begin{aligned}
\int \mathscr{F}^{a c}(\omega, \omega) \frac{\delta_{L} F^{b}(\beta)}{\delta \omega^{c}}+\int \mathscr{F}^{a c}(\omega, \beta) \frac{\delta_{L} F^{b}(\beta)}{\delta \beta^{c}} & +\int \mathscr{F}^{a c}(\omega, \beta) \frac{\delta_{L} F^{b}(\omega)}{\delta \omega^{c}} \\
& +\int F^{c}(\chi) \frac{\delta_{L} \mathscr{F}^{a b}(\omega, \beta)}{\delta \chi^{c}}=0 .
\end{aligned}
$$

The first term vanishes because $\mathscr{F}^{a c}(\omega, \omega)=0$ by the previous argument, the second and the third terms cannot contain a $\beta^{a}$, while the fourth term must contain only one factor of $\beta^{a}$. Since this is impossible, $\mathscr{F}^{a b}(\omega, \beta)$ must also vanish. It follows by a similar argument that $\mathscr{F}^{A B}=0$ when both indices correspond to $\omega_{\mu}^{a}$ or $\beta^{a}$.

So the sum $\mathscr{F}^{A C} \delta_{L} F^{B} / \delta \chi^{C}$ vanishes for all $(A, B)$, and therefore $\mathscr{F}^{A B}=0$ for all pairs $(A, B)$. It follows that $\Gamma_{N, \infty}[\chi, K]$, and hence the quantum effective action, is at most linear in the antisources $K^{A}$, so that the arguments following Eqn. (4.7) can be used towards a proof of renormalizability.

\section{APPENDIX B: RENORMALIZED BRST TRANSFORMATION}

I need to construct a generalized BRST transformation of the fields. This is a nilpotent transformation which affects the Lorentz properties, ghost numbers and global gauge transformation properties of the fields in exactly the same way as $s$ of Eqn.(3.3) and is identical with the latter where it is linear. Let me calculate the generalized nilpotent transformation $s_{R}$ for one field at a time. For the fields which transform linearly under BRST, this is the same as the original $s$,

$$
\begin{array}{rrrr}
s_{R} \bar{\omega}^{a}=-h^{a}, & s_{R} h^{a}=0, & s_{R} \bar{\omega}_{\mu}^{a}=-h_{\mu}^{a}, & s_{R} h_{\mu}^{a}=0, \\
s_{R} n^{a}=\alpha^{a}, & s_{R} \alpha^{a}=0, & s_{R} \bar{\beta}^{a}=\bar{\alpha}^{a}, & s_{R} \bar{\alpha}^{a}=0 .
\end{array}
$$

For the gauge field $A_{\mu}^{a}$ and the associated ghost $\omega^{a}$, I can write

$$
\begin{aligned}
& s_{R} A_{\mu}^{a}=b_{1}^{a b} \partial_{\mu} \omega^{b}+g d_{1}^{a b c} A_{\mu}^{b} \omega^{c}, \\
& s_{R} \omega^{a}=-\frac{1}{2} g d_{2}^{a b c} \omega^{b} \omega^{c} .
\end{aligned}
$$


The nilpotence condition $s_{R}^{2} \omega^{a}=0$ implies

$$
d_{2}^{a b c} d_{2}^{c d e}+d_{2}^{a d c} d_{2}^{c e b}+d_{2}^{a e c} d_{2}^{c b d}=0 .
$$

Therefore $d_{2}^{a b c}$ must be proportional to the structure constants $f^{a b c}$,

$$
d_{2}^{a b c}=\mathscr{Z} f^{a b c},
$$

where $\mathscr{Z}$ is an arbitrary constant. In $s_{R}^{2} A_{\mu}^{a}=0$, the coefficient of $A_{\mu}^{d} \omega^{e} \omega^{c}$ gives

$$
d_{1}^{a b c} d_{1}^{b d e}-d_{1}^{a b e} d_{1}^{b d c}=\mathscr{Z} d_{1}^{a d b} f^{b e c},
$$

which has the unique solution

$$
d_{1}^{a b c}=\mathscr{Z} f^{a b c}
$$

while the coefficient of $\partial_{\mu} \omega^{c} \omega^{d}$ gives

$$
b_{1}^{a b} f^{b c d}=f^{a b d} b_{1}^{b c},
$$

which implies

$$
b_{1}^{a b}=\mathscr{Z} \mathscr{N}_{1} \delta^{a b},
$$

with $\mathscr{N}_{1}$ again an arbitrary constant.

Let me now write the rules for $\beta^{a}$ and $\theta^{a}$,

$$
\begin{aligned}
& s_{R} \beta^{a}=g d_{3}^{a b c} \beta^{b} \omega^{c}, \\
& s_{R} \theta^{a}=-g d_{4}^{a b c} \theta^{b} \omega^{c}-b_{2}^{a b} \beta^{b} .
\end{aligned}
$$

In $s_{R}^{2} \beta^{a}=0$, the coefficient of $\beta^{d} \omega^{e} \omega^{c}$ gives as in Eqn.(B5),

$$
d_{3}^{a b c}=\mathscr{Z} f^{a b c} .
$$

In $s_{R}^{2} \theta^{a}=0$, the coefficient of $\theta^{d} \omega^{e} \omega^{c}$ gives as in Eqn.(B5),

$$
d_{4}^{a b c}=\mathscr{Z} f^{a b c},
$$

while the coefficient of $\beta^{c} \omega^{d}$ gives as in Eqn.(B7),

$$
b_{2}^{a b}=\mathscr{Z} \mathscr{N}_{2} \delta^{a b} \text {. }
$$

For the fields $\omega_{\mu}^{a}$ and $C_{\mu}^{a}$ the rules are

$$
\begin{aligned}
& s_{R} \omega_{\mu}^{a}=-g d_{5}^{a b c} \omega_{\mu}^{a} \omega^{c}+b_{3}^{a b} \partial_{\mu} \beta^{b}+g d_{6}^{a b c} A_{\mu}^{b} \beta^{c}, \\
& s_{R} C_{\mu}^{a}=g d_{7}^{a b c} C_{\mu}^{b} \omega^{c}+b_{4}^{a b} \omega_{\mu}^{b}+b_{5}^{a b} \partial_{\mu} \theta^{b}+g d_{8}^{a b c} A_{\mu}^{b} \theta^{c} .
\end{aligned}
$$

In $s_{R}^{2} \omega_{\mu}^{a}=0$, the coefficient of $\omega_{\mu}^{d} \omega^{e} \omega^{c}$ gives as in Eqn.(B5),

$$
d_{5}^{a b c}=\mathscr{Z} f^{a b c},
$$


the coefficient of $\partial_{\mu} \beta^{c} \omega^{d}$ gives as in Eqn.(B7)

$$
b_{3}^{a b}=\mathscr{Z} \mathscr{N}_{3} \delta^{a b},
$$

with $\mathscr{N}_{3}$ arbitrary, the coefficient of $\beta^{d} \partial_{\mu} \omega^{e}$ gives upon using Eqn.(B15),

$$
d_{6}^{a b c}=\frac{\mathscr{Z} \mathscr{N}_{3}}{\mathscr{N}_{1}} f^{a b c},
$$

and the coefficient of $A_{\mu}^{d} \beta^{e} \omega^{c}$ vanishes identically as a result.

In $s_{R}^{2} C_{\mu}^{a}=0$, the coefficient of $C_{\mu}^{d} \omega^{e} \omega^{c}$ gives as in Eqn.(B5),

$$
d_{7}^{a b c}=\mathscr{Z} f^{a b c},
$$

the coefficients of $\omega_{\mu}^{d} \omega^{c}$ and $\partial_{\mu} \theta^{d} \omega^{c}$ give as in Eqn.(B7),

$$
\begin{gathered}
b_{4}^{a b}=\mathscr{Z} \mathscr{N}_{4} \delta^{a b}, \\
b_{5}^{a b}=\mathscr{Z} \mathscr{N}_{5} \delta^{a b},
\end{gathered}
$$

where $\mathscr{N}_{4}$ and $\mathscr{N}_{5}$ are arbitrary constants. The coefficient of $\partial_{\mu} \beta^{b}$ then gives

$$
\mathscr{N}_{3} \mathscr{N}_{4}=\mathscr{N}_{2} \mathscr{N}_{5}
$$

the coefficient of $A_{\mu}^{b} \beta^{c}$ gives

$$
d_{8}^{a b c}=\frac{\mathscr{Z} \mathscr{N}_{5}}{\mathscr{N}_{1}} f^{a b c}
$$

and the coefficients of $A_{\mu}^{d} \theta^{e} \omega^{c}$ and $\theta^{b} \partial_{\mu} \omega^{c}$ vanish identically as a result.

Finally, $B_{\mu \nu}^{a}$ transforms as

$$
\begin{aligned}
s_{R} B_{\mu \nu}^{a}=g d_{9}^{a b c} B_{\mu \nu}^{b} \omega^{c} & +b_{6}^{a b} \partial_{[\mu} \omega_{\nu]}^{b}+g d_{10}^{a b c} A_{[\mu}^{b} \omega_{\nu]}^{c} \\
& +g d_{11}^{a b c} \partial_{[\mu} A_{\nu]}^{b} \theta^{c}+g^{2} e^{a b c d} A_{\mu}^{b} A_{\nu}^{c} \theta^{d} .
\end{aligned}
$$

The constant $e^{a b c d}$ is antisymmetric in two indices, $e^{a c b d}=-e^{a b c d}$. The coefficient of $B_{\mu \nu}^{d} \omega^{e} \omega^{c}$ in $s_{R}^{2} B_{\mu \nu}^{a}=0$ gives as in Eqn.(B5)

$$
d_{9}^{a b c}=\mathscr{Z} f^{a b c},
$$

the coefficient of $\partial_{[\mu} \omega_{\nu]}^{d} \omega^{c}$ gives as in Eqn.(B7),

$$
b_{6}^{a b}=\mathscr{Z} \mathscr{N}_{6} \delta^{a b},
$$

the coefficient of $\left(\partial_{[\mu} \omega^{d}\right) \omega_{\nu]}^{c}$ gives upon using Eqn.(B23)

$$
d_{10}^{a b c}=\frac{\mathscr{Z} \mathscr{N}_{6}}{\mathscr{N}_{1}} f^{a b c}
$$

the coefficient of $\partial_{[\mu} A_{\nu]}^{c} \beta^{d}$ gives upon using Eqn.(B23) 


$$
d_{11}^{a b c}=\frac{\mathscr{Z} \mathscr{N}_{3} \mathscr{N}_{6}}{\mathscr{N}_{1} \mathscr{N}_{2}} f^{a b c}
$$

the coefficient of $A_{\mu}^{b} \partial_{\nu} \omega^{c} \theta^{d}$ gives upon using Eqn.(B25)

$$
e^{a b c d}=\frac{\mathscr{Z} \mathscr{N}_{3} \mathscr{N}_{6}}{\mathscr{N}_{1}^{2} \mathscr{N}_{2}} f^{a e d} f^{e b c}
$$

All other coefficients in the expression of $s_{R} B_{\mu \nu}^{a}$ vanish identically as a result.

Note that it is possible to consider other terms in $s_{R}$ which obey the Zinn-Justin equation at first order in the antisources. I have ignored such terms because they vanish upon using the symmetry $t$. Let me consider one example, that of $\omega^{a}$. The Zinn-Justin equation says that

$$
s \mathscr{F}^{a}[\omega]-g f^{a b c} \mathscr{F}^{b}[\omega] \omega^{c}=0 .
$$

On the other hand, from Eqn.(4.23) for $\omega^{a}$, I have

$$
t \mathscr{F}^{a}[\omega]=0 .
$$

The only allowed possibility for $\mathscr{F}^{a}[\omega]$ is then

$$
\mathscr{F}^{a}[\omega]=-\frac{1}{2 \epsilon} g\left(d_{2}^{a b c}-f^{a b c}\right) \omega^{b} \omega^{c}+e^{a b c}\left(\omega_{\mu}+D_{\mu} \theta\right)^{b}\left(\omega^{\mu}+D^{\mu} \theta\right)^{c},
$$

where $d_{2}^{a b c}$ and $e^{a b c}$ are now arbitrary. Eqn.(4.24) gives

$$
t \mathscr{F}^{a}[\theta]+2 \mathscr{F}^{a}[\omega]=0,
$$

which immediately shows that $e^{a b c}=0$, and I can write Eqn. (B2) for the transformation of $\omega^{a}$.

Another byproduct of this equation is the somewhat unexpected relation

$$
\mathscr{Z} \mathscr{N}_{2}=1 \text {. }
$$

Similarly, I can use Eqn.(4.23) to relate some of the constants previously found. From $t_{R} s_{R} \omega_{\mu}^{a}=0$, I find $\mathscr{N}_{1}=\mathscr{N}_{3}$, and from $t_{R} s_{R} C_{\mu}^{a}=0$, I find $\mathscr{N}_{5}=\mathscr{Z} \mathscr{N}_{1} \mathscr{N}_{4}$. No other new relation can be found this way.

The transformation rules can now be collected,

$$
\begin{aligned}
& s_{R} A_{\mu}^{a}= \mathscr{Z}\left(\mathscr{N}_{1} \partial_{\mu} \omega^{a}+g f^{a b c} A_{\mu}^{b} \omega^{c}\right), \\
& s_{R} \omega^{a}=-\frac{1}{2} \mathscr{Z} g f^{a b c} \omega^{b} \omega^{c}, \quad s_{R} \bar{\omega}^{a}=-h^{a}, \quad s_{R} h^{a}=0, \\
& s_{R} B_{\mu \nu}^{a}= \mathscr{Z}\left(g f^{a b c} B_{\mu \nu}^{b} \omega^{c}+\mathscr{N}_{6} \partial_{[\mu} \omega_{\nu]}^{a}+\frac{\mathscr{N}_{6}}{\mathscr{N}_{1}} g f^{a b c} A_{[\mu}^{b} \omega_{\nu]}^{c}\right. \\
&\left.\quad \mathscr{Z} \mathscr{N}_{6} g f^{a b c} \partial_{[\mu} A_{\nu]}^{b} \theta^{c}+\frac{\mathscr{Z} \mathscr{N}_{6}}{\mathscr{N}_{1}} g^{2} f^{a e d} f^{e b c} A_{\mu}^{b} A_{\nu}^{c} \theta^{d}\right), \\
& s_{R} C_{\mu}^{a}= \mathscr{Z}\left(g f^{a b c} C_{\mu}^{b} \omega^{c}+\mathscr{N}_{4} \omega_{\mu}^{a}+\mathscr{Z} \mathscr{N}_{1} \mathscr{N}_{4} \partial_{\mu} \theta^{a}+\mathscr{Z} \mathscr{N}_{4} g f^{a b c} A_{\mu}^{b} \theta^{c}\right), \\
& s_{R} \omega_{\mu}^{a}= \mathscr{Z}\left(-g f^{a b c} \omega_{\mu}^{b} \omega^{c}+\mathscr{N}_{1} \partial_{\mu} \beta^{a}+g f^{a b c} A_{\mu}^{b} \beta^{c}\right), \\
& s_{R} \bar{\omega}_{\mu}^{a}=-h_{\mu}^{a}, \quad s_{R} h_{\mu}^{a}=0, \quad s_{R} n^{a}=\alpha^{a}, \quad s_{R} \alpha^{a}=0, \\
& s_{R} \beta^{a}=\mathscr{Z} g f^{a b c} \beta^{b} \omega^{c}, \quad s_{R} \bar{\beta}^{a}=\bar{\alpha}^{a}, \quad s_{R} \bar{\alpha}^{a}=0, \\
& s_{R} \theta^{a}=-\mathscr{Z} g f^{a b c} \theta^{b} \omega^{c}-\beta^{a} .
\end{aligned}
$$




\section{APPENDIX C: DERIVATION OF EQN.(5.6)}

The generic form of the effective action is

$$
\Gamma=\sum_{A} \lambda^{A} X^{A}+\sum_{A, B} \lambda^{A} \lambda^{B} X^{A B}
$$

where $X^{A}$ and $X^{A B}$ do not contain any of the $\lambda^{A}$. Therefore as mentioned in Sec. $\square$ I can write the effect of $s_{R}^{\prime}$ on $\Gamma$ as

$$
\sum_{A}\left(s_{R}^{\prime} \lambda^{A}\right) X^{A}+\sum_{A, B}\left(s_{R}^{\prime} \lambda^{A}\right) \lambda^{B} X^{A B}+\sum_{A, B}(-1)^{\varepsilon_{A}} \lambda^{A}\left(s_{R}^{\prime} \lambda^{B}\right) X^{A B}=0 .
$$

Since $X^{A}$ and $X^{A B}$ do not contain any of the $\lambda^{A}$, I can look at the coefficients of the various $\lambda^{A}$ in the expansion of Eqn.(C2) and set them to zero in order to get an expression for the effective action $\Gamma$. The effect of $s_{R}^{\prime}$ on $\lambda^{A}$ is, for quick reference,

$$
\begin{aligned}
& s_{R}^{\prime} \bar{\omega}^{a}=-\left(h^{a}+\frac{1}{\xi} f^{a}\right), \quad s_{R}^{\prime} \bar{\beta}^{a}=\left(\bar{\alpha}^{a}-\frac{1}{\zeta} \partial_{\mu} \bar{\omega}^{a \mu}\right), \\
& s_{R}^{\prime} \bar{\omega}_{\mu}^{a}=-\left(h_{\mu}^{a}+\frac{1}{\eta} \partial_{\mu} n^{a}+\frac{1}{\eta} f_{\mu}^{a}\right), \quad s_{R}^{\prime} \bar{\alpha}^{a}=-\frac{1}{\zeta} \partial^{\mu}\left(h_{\mu}^{a}+\frac{2}{\eta} \partial_{\mu} n^{a}+\frac{2}{\eta} f_{\mu}^{a}\right), \\
& s_{R}^{\prime} h^{a}=\frac{1}{\xi} \Delta_{R}^{a}, \quad s_{R}^{\prime} \alpha^{a}=\frac{1}{\zeta} \Delta_{R}^{\prime a}, \\
& s_{R}^{\prime} h_{\mu}^{a}=-\frac{1}{\eta}\left(\partial_{\mu} \alpha^{a}+\frac{2}{\zeta} \partial_{\mu} f^{\prime a}-\Delta_{R \mu}^{a}\right), \quad s_{R}^{\prime} n^{a}=\left(\alpha^{a}+\frac{1}{\zeta} f^{\prime a}\right) .
\end{aligned}
$$

In this Appendix, I will construct the most general $\Gamma$ obeying Eqn. (C2). For each $\lambda^{A} \mathrm{I}$ will first consider coefficients of terms containing $\lambda^{A} \lambda^{B}$ in the expansion of Eqn.(C2). There can be no term of third or higher order in $\lambda^{A}$ in the effective action because of the constant shift symmetries, and therefore the left hand side of Eqn.(C2) can be at most quadratic in the $\lambda^{A}$. The coefficients will have as many ghost fields and derivative operators as necessary. Setting the coefficients to zero will eliminate some of the terms from the effective action and produce relations among some others. Following the same procedure for the terms linear in $\lambda^{A}$ will produce some more relations. There will also be a few terms not containing any power of $\lambda^{A}$ in the expansion of Eqn. (C2). The sum of these should also vanish.

I will consider $\lambda^{A}$ in the order $\left(\bar{\omega}^{a}, \bar{\beta}^{a}, \bar{\omega}_{\mu}^{a}, \bar{\alpha}^{a}, h^{a}, \alpha^{a}, h_{\mu}^{a}, \partial_{\mu} n^{a}\right)$. Terms containing products of the form $\bar{\omega}^{a} \lambda^{B}$ in the expansion of $s_{R}^{\prime} \Gamma$ come from the $s_{R^{\prime}}^{\prime}$ variation of

$$
\bar{\omega}^{a} \bar{\omega}^{b} X_{\bar{\omega} \bar{\omega}}^{a b}+\bar{\omega}^{a} \bar{\beta}^{b} X_{\bar{\omega} \bar{\beta}}^{a b}+\bar{\omega}^{a} \bar{\alpha}^{b} X_{\bar{\omega} \bar{\alpha}}^{a b}+\bar{\omega}^{a} \bar{\omega}_{\mu}^{b} X_{\bar{\omega} \bar{\omega}_{*}}^{a b \mu}+\bar{\omega}^{a} h_{\mu}^{b} X_{\bar{\omega} h_{*}}^{a b \mu}+\bar{\omega}^{a} \partial_{\mu} n^{b} X_{\bar{\omega} n}^{a b \mu},
$$

where the subscripts $\bar{\omega} \bar{\omega}$ etc. indicate the quadratic combination which couples to a given $X$, and an asterisk indicates the presence of a Lorentz index on the subscript. In the first term, $X_{\bar{\omega} \bar{\omega}}^{a b}$ has to be antisymmetric in $[a, b]$. Therefore, the coefficient of $\bar{\omega}^{a} h^{b}$ in the expansion of $s_{R}^{\prime} \Gamma$ gives $X_{\bar{\omega} \bar{\omega}}^{a b}=0$. The coefficients of $\bar{\omega}^{a} \bar{\alpha}^{b}, \bar{\omega}^{a} h_{\mu}^{b}$ and $\bar{\omega}^{a} \partial_{\mu} n^{b}$ give

$$
X_{\bar{\omega} \bar{\beta}}^{a b}=X_{\bar{\omega} \bar{\alpha}}^{a b}=X_{\bar{\omega} \bar{\omega}_{*}}^{a b \mu}=0
$$


while the coefficient of $\bar{\omega}^{a} \alpha^{b}$ gives $X_{\bar{\omega} h_{*}}^{a b \mu}=\eta X_{\bar{\omega} n}^{a b \mu}$. Terms linear in $\bar{\omega}^{a}$ (and containing no other $\lambda^{A}$ ) come from

$$
\bar{\omega}^{a} \alpha^{b} X_{\bar{\omega} \alpha}^{a b}+\bar{\omega}^{a} h^{b} X_{\bar{\omega} h}^{a b}+\left(\eta \bar{\omega}^{a} h_{\mu}^{b}+\bar{\omega}^{a} \partial_{\mu} n^{b}\right) X_{\bar{\omega} n}^{a b \mu}
$$

The coefficient of $\bar{\omega}^{a}$ in $s_{R}^{\prime} \Gamma$ is therefore

$$
-\frac{1}{\zeta} \Delta_{R}^{\prime b} X_{\bar{\omega} \alpha}^{a b}-\frac{1}{\xi} \Delta_{R}^{b} X_{\bar{\omega} h}^{a b}+\left(\frac{1}{\zeta} \partial_{\mu} f^{\prime b}-\Delta_{R \mu}^{b}\right) X_{\bar{\omega} n}^{a b \mu}=0 .
$$

Each of the $X$ 's in this equation must contain at least one derivative operator to allow for the constant shift symmetry of $\bar{\omega}^{a}$. Therefore, the $X$ 's must be constructed only out of fields of mass dimension zero. Since the factors multiplying the $X$ 's are all different, and have fields of non-vanishing mass dimension, the only choice for which this equation can be satisfied is

$$
X_{\bar{\omega} \alpha}^{a b}=X_{\bar{\omega} h}^{a b}=X_{\bar{\omega} n}^{a b \mu}=X_{\bar{\omega} h_{*}}^{a b \mu}=0 .
$$

Thus, all terms containing $\bar{\omega}^{a} \lambda^{B}$ are excluded from the effective action.

Terms containing $\bar{\beta}^{a} \lambda^{B}$ arise from the terms

$$
\bar{\beta}^{a} \bar{\beta}^{b} X_{\bar{\beta} \bar{\beta}}^{a b}+\bar{\beta}^{a} \bar{\omega}_{\mu}^{b} X_{\bar{\beta} \bar{\omega}_{*}}^{a b}+\bar{\beta}^{a} \bar{\alpha}^{b} X_{\bar{\beta} \bar{\alpha}}^{a b}+\bar{\beta}^{a} h_{\mu}^{b} X_{\bar{\beta} h_{*}}^{a b \mu}+\bar{\beta}^{a} \partial_{\mu} n^{b} X_{\bar{\beta} n}^{a b \mu}
$$

As before, I set the coefficients of the quadratic terms in the expansion to zero. The coefficients of $\bar{\beta}^{a} \bar{\alpha}^{b}, \bar{\beta}^{a} h_{\mu}^{b}$ and $\bar{\beta}^{a} \partial_{\mu} n^{b}$ give

$$
X_{\bar{\beta} \bar{\beta}}^{a b}=X_{\bar{\beta} \bar{\omega}_{*}}^{a b}=X_{\bar{\beta} \bar{\alpha}}^{a b}=0,
$$

while the coefficient of $\bar{\beta}^{a} \bar{\alpha}^{b}$ gives $X_{\bar{\beta} h_{*}}^{a b \mu}=\eta X_{\bar{\beta} n}^{a b \mu}$.

Terms linear in $\bar{\beta}^{a}$ appear from the $s_{R}^{\prime}$-variation of

$$
\bar{\beta}^{a} h^{b} X_{\bar{\beta} h}^{a b}+\bar{\beta}^{a} \alpha^{b} X_{\bar{\beta} \alpha}^{a b}-\left(\eta \bar{\beta}^{a} h_{\mu}^{b}+\bar{\beta}^{a} \partial_{\mu} n^{b}\right) X_{\bar{\beta} n}^{a b \mu} .
$$

The coefficient of $\bar{\beta}^{a}$ in the variation of this is

$$
\frac{1}{\xi} \Delta_{R}^{b} X_{\bar{\beta} h}^{a b}+\frac{1}{\zeta} \Delta_{R}^{\prime b} X_{\bar{\beta} \alpha}^{a b}-\left(\frac{1}{\zeta} \partial_{\mu} f^{\prime b}-\Delta_{R \mu}^{b}\right) X_{\bar{\beta} n}^{a b \mu}=0 .
$$

Again each of the $X$ 's in this equation has mass dimension zero after excluding the derivative operator they must contain to allow a constant shift in $\bar{\beta}^{a}$. It is easy to see that the only solution is

$$
X_{\bar{\beta} h}^{a b}=X_{\bar{\beta} \alpha}^{a b}=X_{\bar{\beta} n}^{a b \mu}=X_{\bar{\beta} h_{*}}^{a b \mu}=0 .
$$

This, together with the previous result rules out all terms containing $\bar{\beta}^{a} \lambda^{B}$.

Next are the terms containing $\bar{\omega}_{\mu}^{a} \lambda^{B}$, which come from the variation of

$$
\bar{\omega}_{\mu}^{a} \bar{\omega}_{\nu}^{b} X_{\bar{\omega}_{*} \bar{\omega}_{*}}^{a b \mu \nu}+\bar{\omega}_{\mu}^{a} \bar{\alpha}^{b} X_{\bar{\omega}_{*} \bar{\alpha}}^{a b \mu}+\bar{\omega}_{\mu}^{a} h_{\nu}^{b} X_{\bar{\omega}_{*} h_{*}}^{a b \mu \nu}+\bar{\omega}_{\mu}^{a} \partial_{\nu} n^{b} X_{\bar{\omega}_{*} n}^{a b \mu \nu}
$$




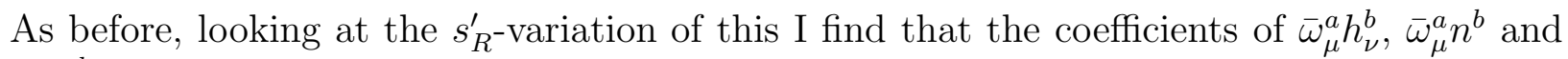
$\bar{\omega}_{\mu}^{a} \alpha^{b}$ imply

$$
X_{\bar{\omega}_{*} \bar{\omega}_{*}}^{a b \mu \nu}=X_{\bar{\omega}_{*} \bar{\alpha}}^{a b \mu}=0, \quad X_{\bar{\omega}_{*} h_{*}}^{a b \mu \nu}=\eta X_{\bar{\omega}_{*} n}^{a b \mu \nu}
$$

Terms linear in $\bar{\omega}_{\mu}^{a}$ come from varying

$$
\bar{\beta}^{a} X_{\bar{\beta}}^{a}+\bar{\omega}_{\mu}^{a} h^{b} X_{\bar{\omega}_{*} h}^{a b \mu}+\bar{\omega}_{\mu}^{a} \alpha^{b} X_{\bar{\omega}_{*} \alpha}^{a b \mu}+\bar{\omega}_{\mu}^{a}\left(\eta h_{\nu}^{b}+\partial_{\nu} n^{b}\right) X_{\bar{\omega}_{*} n}^{a b \mu \nu} .
$$

The coefficient of $\bar{\omega}_{\nu}^{a}$, or more precisely the functional derivative $\delta_{L} / \delta \bar{\omega}_{\mu}^{a}$, in the $s_{R}^{\prime}$-variation of this gives the relation

$$
\frac{1}{\zeta} \partial^{\mu} X_{\bar{\beta}}^{a}-\frac{1}{\xi} \Delta_{R}^{b} X_{\bar{\omega}_{* h}}^{a b \mu}-\frac{1}{\zeta} \Delta_{R}^{\prime b} X_{\bar{\omega}_{*} \alpha}^{a b \mu}+\left(\frac{1}{\zeta} \partial_{\nu} f^{\prime b}-\Delta_{R \nu}^{b}\right) X_{\bar{\omega}_{*} n}^{a b \mu \nu}=0 .
$$

This equation contains $X_{\bar{\beta}}^{a}$ which has non-vanishing mass dimension, so it an contain fields other than $\theta^{a}$ and $C_{\mu}^{a}$, and the argument used in previous cases cannot be applied here. Therefore, the $X$ 's appearing here must remain undetermined for the moment.

Terms containing products of the form $\bar{\alpha}^{a} \lambda^{B}$ appear in the $s_{R^{\prime} \text {-variation of }}$

$$
\bar{\alpha}^{a} \bar{\alpha}^{b} X_{\bar{\alpha} \bar{\alpha}}^{a b}+\bar{\alpha}^{a} h_{\mu}^{b} X_{\bar{\alpha} h_{*}}^{a b \mu}+\bar{\alpha}^{a} \partial_{\mu} n^{b} X_{\bar{\alpha} n}^{a b \mu}
$$

The coefficients of $\bar{\alpha}^{a} h_{\mu}^{b}$ and $\bar{\alpha}^{a} \alpha^{b}$ in the variation give

$$
X_{\bar{\alpha} \bar{\alpha}}^{a b}=0, \quad X_{\bar{\alpha} h_{*}}^{a b \mu}=\eta X_{\bar{\alpha} n}^{a b \mu} .
$$

Terms linear in $\bar{\alpha}^{a}$ come from

$$
\bar{\beta}^{a} X_{\bar{\beta}}^{a}-\bar{\alpha}^{a} h^{b} X_{\bar{\alpha} h}^{a b}+\bar{\alpha}^{a} \alpha^{b} X_{\bar{\alpha} \alpha}^{a b}+\bar{\alpha}^{a}\left(\eta h_{\mu}^{b}+\partial_{\mu} n^{b}\right) X_{\bar{\alpha} n}^{a b \mu} .
$$

The coefficient of $\bar{\alpha}^{a}$ in the $s_{R}^{\prime}$-variation of this satisfies

$$
X_{\bar{\beta}}^{a}-\frac{1}{\xi} \Delta_{R}^{b} X_{\bar{\alpha} h}^{a b}-\frac{1}{\zeta} \Delta_{R}^{\prime b} X_{\bar{\alpha} \alpha}^{a b}+\left(\partial_{\mu} f^{\prime b}-\Delta_{R \mu}^{b}\right) X_{\bar{\alpha} n}^{a b \mu}=0 .
$$

This equation is again insufficient to determine the $X$ 's in it and will have to be reexamined later.

Terms containing $h^{a} \lambda^{B}$ come from the variation of

$$
\bar{\omega}_{\mu}^{a} h^{b} X_{\bar{\omega}_{*} h}^{a b \mu}+\bar{\alpha}^{a} h^{b} X_{\bar{\alpha} h}^{a b}+h^{a} h_{\mu}^{b} X_{h h_{*}}^{a b \mu}+h^{a} \partial_{\mu} n^{b} X_{h n}^{a b \mu},
$$

the other possibilities being known to vanish from the above analysis. The coefficients of $h^{a} h_{\mu}^{b}, h^{a} n^{b}$ and $h^{a} \alpha^{b}$ in the variation of this lead to

$$
X_{\bar{\omega}_{*} h}^{a b \mu}=X_{\bar{\alpha} h}^{a b}=0, \quad X_{h h_{*}}^{a b \mu}=\eta X_{h n}^{a b \mu} .
$$

Terms linear in $h^{a}$ come from

$$
\bar{\omega}^{a} X_{\bar{\omega}}^{a}+h^{a} h^{b} X_{h h}^{a b}+h^{a} \alpha^{b} X_{h \alpha}^{a b}+h^{a}\left(\eta h_{\mu}^{b}+\partial_{\mu} n^{b}\right) X_{h n}^{a b \mu} .
$$


Of these, $X_{h \alpha}^{a b}$ has vanishing mass dimension and ghost number -1 . Since it is not possible to construct such a function with the fields in the theory, it follows that $X_{h \alpha}^{a b}=0$. The coefficient of $h^{a}$, in the terms linear in $h^{a}$, in the variation of the rest satisfies the equation

$$
-X_{\bar{\omega}}^{a}+\frac{2}{\xi} \Delta_{R}^{b} X_{h h}^{a b}-\left(\frac{1}{\zeta} \partial_{\mu} f^{\prime b}-\Delta_{R \mu}^{b}\right) X_{h n}^{a b \mu}=0 .
$$

These terms will also be left for later scrutiny, as this equation is insufficient to determine them.

Terms containing $\alpha^{a} \lambda^{B}$ come from the $s_{R^{-v a r i a t i o n ~ o f ~}}^{\prime}$

$$
\bar{\omega}_{\mu}^{a} \alpha^{b} X_{\bar{\omega}_{*} \alpha}^{a b \mu}+\bar{\alpha}^{a} \alpha^{b} X_{\bar{\alpha} \alpha}^{a b}+h_{\mu}^{a} h_{\nu}^{b} X_{h_{*} h_{*}}^{a b b \nu}+h_{\mu}^{a} \partial_{\nu} n^{b} X_{h_{*} n}^{a b \mu \nu}+\partial_{\mu} n^{a} \partial_{\nu} n^{b} X_{n n}^{a b \mu \nu},
$$

where I have excluded terms that have already been shown to vanish, and set

$$
X_{h \alpha}^{a b}=X_{\alpha h_{*}}^{a b \mu}=X_{\alpha n}^{a b \mu}=X_{\alpha \alpha}^{a b}=0,
$$

because these $X$ 's have negative ghost number and vanishing mass dimension, so cannot be constructed out of fields present in the theory. The terms containing products of the form $\alpha^{a} \lambda^{B}$ in the $s_{R^{\prime}}^{\prime}$-variation of of this can be set to zero to give the equation

$$
\begin{aligned}
-\left(h_{\mu}^{a}+\frac{1}{\eta} \partial_{\mu} n^{a}\right) \alpha^{b} X_{\bar{\omega}_{*} \alpha}^{a b \mu} & -\frac{1}{\zeta} \partial_{\mu}\left(h_{\mu}^{a}+\frac{2}{\eta} \partial_{\mu} n^{a}\right) \alpha^{b} X_{\bar{\alpha} \alpha}^{a b}-\frac{2}{\eta} \partial_{\mu} \alpha^{a} h_{\nu}^{b} X_{h_{*} h_{*}}^{a b \mu \nu} \\
& -\frac{1}{\eta} \partial_{\mu} \alpha^{a} \partial_{\nu} n^{b} X_{h_{*} n}^{a b \mu \nu}+h_{\mu}^{a} \partial_{\nu} \alpha^{b} X_{h_{*} n}^{a b \mu \nu}+2 \partial_{\mu} \alpha^{a} \partial_{\nu} n^{b} X_{n n}^{a b \mu \nu}=0
\end{aligned}
$$

where I have used the fact that both $X_{h_{*} h_{*}}^{a b \mu \nu}$ and $X_{n n}^{a b \mu \nu}$ are symmetric under the exchange $[\mu, a] \leftrightarrow[\nu, b]$. This equation will also be put aside for later use. Terms linear in $\alpha^{a}$ come from

$$
\bar{\omega}_{\mu}^{a} \alpha^{b} X_{\bar{\omega}_{*} \alpha}^{a b \mu}+\bar{\alpha}^{a} \alpha^{b} X_{\bar{\alpha} \alpha}^{a b}+h_{\mu}^{a} X_{h_{*}}^{a \mu}+\partial_{\mu} n^{a} X_{n}^{a \mu}+\alpha^{a} X_{\alpha}^{a} .
$$

Of these, $X_{\alpha}^{a}$ has ghost number -1 , and must satisfy $s_{R}^{\prime} X_{\alpha}^{a}=0$ by definition (Eqn.(5.4)), which cannot happen unless $X_{\alpha}^{a}=0$. The terms linear in $\alpha^{a}$ in the $s_{R}^{\prime}$-variation of the rest lead to the equation

$$
-\frac{1}{\eta} f_{\mu}^{b} X_{\bar{\omega}_{*} \alpha}^{b a \mu}-\frac{2}{\zeta \eta} \partial_{\mu} f^{b \mu} X_{\bar{\alpha} \alpha}^{a b}+\frac{1}{\eta} \partial_{\mu} X_{h_{*}}^{a \mu}-\partial_{\mu} X_{n}^{a \mu}=0 .
$$

The terms containing $h_{\mu}^{a} \lambda^{B}$ come from the $s_{R}^{\prime}$-variation of

$$
\begin{aligned}
\bar{\omega}_{\mu}^{a} h^{b} X_{\bar{\omega}_{*} h}^{a b \mu} & +\bar{\omega}_{\mu}^{a} \alpha^{b} X_{\bar{\omega}_{*} \alpha}^{a b \mu}+\left(\eta \bar{\omega}_{\mu}^{a} h_{\nu}^{b}+\bar{\omega}_{\mu}^{a} \partial_{\nu} n^{b}\right) X_{\bar{\omega}_{* n}}^{a b \mu \nu}+\bar{\alpha}^{a} \alpha^{b} X_{\bar{\alpha} \alpha}^{a b} \\
& +\left(\eta \bar{\alpha}^{a} h_{\mu}^{b}+\bar{\alpha}^{a} \partial_{\mu} n^{b}\right) X_{\bar{\alpha} n}^{a b \mu}+h_{\mu}^{a} h_{\nu}^{b} X_{h_{*} h_{*}}^{a b \mu \nu}+h_{\mu}^{a} \partial_{\nu} n^{b} X_{h_{*} n}^{a b \mu \nu} .
\end{aligned}
$$

The coefficient of $h_{\mu}^{a} h^{b}$ in the variation of this gives $X_{\bar{\omega}_{*} h}^{a b \mu}=0$, and the remaining terms with $h_{\mu}^{a} \lambda^{B}$ in them satisfy 


$$
\begin{aligned}
-h_{\mu}^{a} \alpha^{b} X_{\bar{\omega}_{*} \alpha}^{a b \mu} & -\left(\eta h_{\mu}^{a} h_{\nu}^{b}+2 h_{\mu}^{a} \partial_{\nu} n^{b}\right) X_{\bar{\omega}_{*} n}^{a b \mu \nu}-\frac{1}{\zeta} \partial_{\mu} h^{a \mu} \alpha^{b} X_{\bar{\alpha} \alpha}^{a b}-\frac{2}{\eta} \partial_{\mu} \alpha^{a} h_{\nu}^{b} X_{h_{*} h_{*}}^{a b \mu \nu} \\
& -\left[\frac{\eta}{\zeta} \partial_{\nu}\left(h^{a \nu}+\frac{2}{\eta} \partial^{\nu} n^{a}\right) h_{\mu}^{b}+\frac{1}{\zeta} \partial_{\nu} h^{a \nu} \partial_{\mu} n^{b}\right] X_{\bar{\alpha} n}^{a b \mu}+h_{\mu}^{a} \partial_{\nu} \alpha^{b} X_{h_{*} n}^{a b \mu \nu}=0 .
\end{aligned}
$$

It follows from this that $X_{\bar{\omega}_{* n} n}^{a b \mu \nu}=X_{\bar{\alpha} n}^{a b \mu}=0$ (essentially because there is no linear combination of $b a^{a}$ and $\partial_{\mu} \bar{\omega}_{\nu}^{a}$ which is $s_{R}^{\prime}$-invariant). Then the remaining terms satisfy

$$
-h_{\mu}^{a} \alpha^{b} X_{\bar{\omega}_{*} \alpha}^{a b \mu}-\frac{1}{\zeta} \partial_{\mu} h^{a \mu} \alpha^{b} X_{\bar{\alpha} \alpha}^{a b}-\frac{2}{\eta} \partial_{\mu} \alpha^{a} h_{\nu}^{b} X_{h_{*} h_{*}}^{a b \mu \nu}+h_{\mu}^{a} \partial_{\nu} \alpha^{b} X_{h_{*} n}^{a b \mu \nu}=0 .
$$

Terms linear in $h_{\mu}^{a}$ appear from the $s_{R}^{\prime}$-variation of

$$
\bar{\omega}_{\mu}^{a} X_{\bar{\omega}_{*}}^{a \mu}+\bar{\alpha}^{a} X_{\bar{\alpha}}^{a}+h^{a}\left(\eta h_{\mu}^{b}+\partial_{\mu} n^{b}\right) X_{h n}^{a b \mu}+h_{\mu}^{a} h_{\nu}^{b} X_{h_{*} h_{*}}^{a b \mu \nu}+h_{\mu}^{a} \partial_{\nu} n^{b} X_{h_{*} n}^{a b \mu \nu},
$$

giving the following equation:

$$
-X_{\bar{\omega}_{*}}^{a \mu}+\frac{1}{\zeta} \partial^{\mu} X_{\bar{\alpha}}^{a}+\frac{\eta}{\xi} \Delta_{R}^{b} X_{h n}^{b a \mu}-\frac{4}{\zeta \eta} \partial_{\nu} f^{\prime b} X_{h_{*} h_{*}}^{a b \mu \nu}+\frac{2}{\eta} \Delta_{R \nu}^{b} X_{h_{*} h_{*}}^{a b \mu \nu}+\frac{1}{\zeta} \partial_{\nu} f^{\prime b} X_{h_{*} n}^{a b \mu \nu}=0 .
$$

Finally, terms containing $\partial_{\mu} n^{a} \lambda^{B}$ come from the variation of

$$
\bar{\omega}_{\mu}^{a} \alpha^{b} X_{\bar{\omega}_{*} \alpha}^{a b \mu}+\bar{\alpha}^{a} \alpha^{b} X_{\bar{\alpha} \alpha}^{a b}+h_{\mu}^{a} \partial_{\nu} n^{b} X_{h_{*} n}^{a b \mu \nu}+\partial_{\mu} n^{a} \partial_{\nu} n^{b} X_{n n}^{a b \mu \nu},
$$

and satisfy the equation

$$
-\frac{1}{\eta} \partial_{\mu} n^{a} \alpha^{b} X_{\bar{\omega}_{*} \alpha}^{a b \mu}-\frac{2}{\zeta \eta} \square n^{a} \alpha^{b} X_{\bar{\alpha} \alpha}^{a b}--\frac{1}{\eta} \partial_{\mu} \alpha^{a} \partial_{\nu} n^{b} X_{h_{*} n}^{a b \mu \nu}+2 \partial_{\mu} n^{a} \partial_{\nu} \alpha^{b} X_{n n}^{a b \mu \nu}=0,
$$

while terms linear in $n^{a}$ appear from the variation of

$$
\bar{\omega}_{\mu}^{a} X_{\bar{\omega}_{*}}^{a \mu}+\bar{\alpha}^{a} X_{\bar{\alpha}}^{a}+h^{a} \partial_{\mu} n^{b} X_{h n}^{a b \mu}+h_{\mu}^{a} \partial_{\nu} n^{b} X_{h_{*} n}^{a b \mu \nu}+\partial_{\mu} n^{a} \partial_{\nu} n^{b} X_{n n}^{a b \mu \nu},
$$

and gives the equation

$$
\begin{aligned}
-\frac{1}{\eta} \partial_{\mu} n^{a} X_{\bar{\omega}_{*}}^{a \mu} & -\frac{2}{\zeta \eta} \square n^{a} X_{\bar{\alpha}}^{a}+\frac{1}{\xi} \Delta_{R}^{a} \partial_{\mu} n^{b} X_{h n}^{a b \mu} \\
& -\frac{2}{\zeta \eta} \partial_{\mu} f^{\prime a} \partial_{\nu} n^{b} X_{h_{*} n}^{a b \mu \nu}+\frac{1}{\eta} \Delta_{R \mu}^{a} \partial_{\nu} n^{b} X_{h_{*} n}^{a b \mu \nu}+\frac{2}{\zeta} \partial_{\mu} f^{\prime a} \partial_{\nu} n^{b} X_{n n}^{a b \mu \nu}=0 .
\end{aligned}
$$

There is one more equation that can be obtained from $s_{R}^{\prime} \Gamma=0$, the one involving terms which do not contain any of the $\lambda^{A}$. This equation is

$$
\begin{aligned}
-\frac{1}{\xi} f^{a} X_{\bar{\omega}}^{a}-\frac{1}{\eta} f_{\mu}^{a} X_{\bar{\omega}_{*}}^{a \mu} & -\frac{2}{\zeta \eta} \partial_{\mu} f_{\mu}^{a} X_{\bar{\alpha}}^{a}+\frac{1}{\xi} \Delta_{R}^{a} X_{h}^{a} \\
& -\frac{2}{\zeta \eta} \partial_{\mu} f^{\prime a} X_{h_{*}}^{a \mu}+\frac{1}{\eta} \Delta_{R \mu}^{a} X_{h_{*}}^{a \mu}+\frac{1}{\zeta} \partial_{\mu} f^{\prime a} X_{n}^{a \mu}=0 .
\end{aligned}
$$

I can now write the effective Lagrangian for the ghost sector of the theory, after setting to zero all the $X$ 's that were found to vanish in the analysis so far,

$$
\begin{aligned}
\mathscr{L}_{g}=\bar{\omega}^{a} X_{\bar{\omega}}^{a} & +\bar{\beta}^{a} X_{\bar{\beta}}^{a}+\bar{\omega}_{\mu}^{a} X_{\bar{\omega}_{*}}^{a \mu}+\bar{\alpha}^{a} X_{\bar{\alpha}}^{a}+h^{a} X_{h}^{a}+h_{\mu}^{a} X_{h_{*}}^{a \mu}+\partial_{\mu} n^{a} X_{n}^{a \mu} \\
& +\bar{\omega}_{\mu}^{a} \alpha^{b} X_{\bar{\omega}_{*} \alpha}^{a b \mu}+\bar{\alpha}^{a} \alpha^{b} X_{\bar{\alpha} \alpha}^{a b}+h^{a} h^{b} X_{h h}^{a b}+h^{a}\left(\eta h_{\mu}^{b}+\partial_{\mu} n^{b}\right) X_{h n}^{a b \mu} \\
& +h_{\mu}^{a} h_{\nu}^{b} X_{h_{*} h_{*}}^{a b \mu \nu}+h_{\mu}^{a} \partial_{\nu} n^{b} X_{h_{*} n}^{a b \mu \nu}+\partial_{\mu} n^{a} \partial_{\nu} n^{b} X_{n n}^{a b \mu \nu} .
\end{aligned}
$$




\section{APPENDIX D: DERIVATION OF EQN. (5.8)}

In this Appendix, I shall try to calculate the functions which remained undetermined in Eqn. (5.6) (equivalently, Eqn. (C41)). I start from the expression for the $s_{R}$-variation of $\Gamma$, which can be written as

$$
\begin{aligned}
s_{R} \Gamma= & \sum_{A}\left[\left(s_{R} \lambda^{A}\right) X^{A}+(-1)^{\varepsilon_{A}} \lambda^{A}\left(s_{R} X^{A}\right)\right] \\
& +\sum_{A B}\left[\left(s_{R} \lambda^{A}\right) \lambda^{B} X^{A B}+(-1)^{\varepsilon_{A}} \lambda^{A}\left(s_{R} \lambda^{B}\right) X^{A B}+(-1)^{\varepsilon_{A}+\varepsilon_{B}} \lambda^{A} \lambda^{B}\left(s_{R} X^{A B}\right)\right]=0 .
\end{aligned}
$$

Since $s_{R} X^{A}$ and $s_{R} X^{A B}$ do not contain any of the $\lambda^{A}$, I can consider the coefficients of $\lambda^{A}$ or of $\lambda^{A} \lambda^{B}$ in the above expression and set them to zero one at a time. I will first set to zero the coefficients of $\lambda^{A} \lambda^{B}$, and then the terms linear in $\lambda^{A}$.

The coefficient of $\partial_{\mu} n^{a} \partial_{\nu} \alpha^{b}$ and $h^{a} \partial_{\mu} \alpha^{b}$ give

$$
X_{n n}^{a b \mu \nu}=X_{h n}^{a b \mu}=0,
$$

while the coefficients of $\bar{\alpha}^{a} \alpha^{b}, h^{a} h^{b}, h_{\mu}^{a} h_{\nu}^{b}$ and $h_{\mu}^{a} \partial_{\nu} n^{b}$ give

$$
s_{R} X_{\bar{\alpha} \alpha}^{a b}=s_{R} X_{h h}^{a b}=s_{R} X_{h_{*} h_{*}}^{a b \mu \nu}=s_{R} X_{h_{*} n}^{a b \mu \nu}=0 .
$$

Now, each of the $X$ 's in this equation has zero mass dimension, zero ghost number and is $s_{R}$-invariant, so each must be a (possibly different) constant. Let me define four constants $K_{1}, K_{2}, K_{3}$ and $K_{4}$ as

$$
X_{\bar{\alpha} \alpha}^{a b}=K_{1} \delta^{a b}, \quad X_{h h}^{a b}=K_{2} \delta^{a b}, \quad X_{h_{*} h_{*}}^{a b \mu \nu}=K_{3} g^{\mu \nu} \delta^{a b}, \quad X_{h_{*} n}^{a b \mu \nu}=K_{4}^{\mu \nu} \delta^{a b} .
$$

The coefficients of $h^{a}, \bar{\alpha}^{a}, h_{\mu}^{a}, \alpha^{a}, \bar{\omega}^{a}, \bar{\beta}^{a}, \bar{\omega}_{\mu}^{a}$ and $n^{a}$ give the equations

$$
\begin{aligned}
-X_{\bar{\omega}}^{a}+s_{R} X_{h}^{a} & =0, \\
X_{\bar{\beta}}^{a}-s_{R} X_{\bar{\alpha}}^{a} & =0, \\
-X_{\bar{\omega}_{*}}^{a \mu}+s_{R} X_{h_{*}}^{a \mu} & =0, \\
\partial_{\mu} X_{n}^{a \mu} & =0, \\
s_{R} X_{\bar{\omega}}^{a}=s_{R} X_{\bar{\beta}}^{a}=s_{R} X_{\bar{\omega}_{*}}^{a} & =s_{R} \partial_{\mu} X_{n}^{a \mu}=0 .
\end{aligned}
$$

The last equation in this list is redundant as it can be obtained by applying $s_{R}$ to the previous equations and remembering that $s_{R}^{2}=0$. The coefficient of $\bar{\omega}_{\mu}^{a} \alpha^{b}$ gives $s_{R} X_{\bar{\omega}_{*} \alpha}^{a b \mu}=0$, but $X_{\bar{\omega}_{* \alpha}}^{a b \mu}$ must contain a derivative operator to allow for the constant shift symmetry of $\bar{\omega}_{\mu}^{a}$. So as with the functions in Eqn.(D3), $X_{\bar{\omega}_{*} \alpha}^{a b \mu}$ must be a constant times a derivative operator. The coefficient of $h_{\mu}^{a} \alpha^{b}$ in $s_{R} \mathscr{L}_{g}$ shows that this constant is $K_{4}^{\mu \nu}$, so I can write the ghost sector Lagrangian as

$$
\begin{aligned}
\mathscr{L}_{g}=\bar{\omega}^{a} X_{\bar{\omega}}^{a} & +\bar{\beta}^{a} X_{\bar{\beta}}^{a}+\bar{\omega}_{\mu}^{a} X_{\bar{\omega}_{*}}^{a \mu}+\bar{\alpha}^{a} X_{\bar{\alpha}}^{a}+h^{a} X_{h}^{a}+h_{\mu}^{a} X_{h_{*}}^{a \mu} \\
& -K_{4}^{\mu \nu} \partial_{\nu} \bar{\omega}_{\mu}^{a} \alpha^{a}+K_{1} \bar{\alpha}^{a} \alpha^{a}+K_{2} h^{a} h^{a}+K_{3} h_{\mu}^{a} h_{\mu}^{a}+K_{4}^{\mu \nu} h_{\mu}^{a} \partial_{\nu} n^{a} .
\end{aligned}
$$

Now I can use the unused equations from Appendix C. These were Eqs. (C17), (C21), (C25), (C28), (C30), (C35), and (C40). 
Eqn. (C21) now reads

$$
X_{\bar{\beta}}^{a}-\frac{K_{1}}{\zeta} \Delta_{R}^{\prime a}=0 .
$$

In keeping with standard notation, let me rewrite

$$
K_{1}=\zeta Z_{\beta} \quad \Rightarrow \quad X_{\bar{\beta}}^{a}=Z_{\beta} \Delta_{R}^{\prime a} .
$$

Eqn.(C25) now becomes

$$
-X_{\bar{\omega}}^{a}+\frac{2}{\xi} K_{2} \Delta_{R}^{a}=0 .
$$

As in the above, let me redefine the constant,

$$
K_{2}=\frac{\xi}{2} Z_{\omega} \quad \Rightarrow \quad X_{\bar{\omega}}^{a}=Z_{\omega} \Delta_{R}^{a}
$$

Eqn. (C17) becomes

$$
\frac{1}{\zeta} \partial^{\mu} X_{\bar{\beta}}^{a}-\frac{1}{\zeta} K_{4}^{\mu \nu} \partial_{\nu} \Delta_{R}^{\prime a}=0,
$$

which gives upon using Eqn.(D8) that

$$
K_{4}^{\mu \nu}=Z_{\beta} g^{\mu \nu} .
$$

Using this and Eqn.(D8) I can rewrite Eqn.(C33) as

$$
Z_{\beta} \partial_{\mu} h^{a \mu} \alpha^{a}-Z_{\beta} \partial_{\mu} h^{a \mu} \alpha^{a}-\frac{2}{\eta} K_{3} h^{a \mu} \partial_{\mu} \alpha^{a}+Z_{\beta} h^{a \mu} \partial_{\mu} \alpha^{a}=0,
$$

from which it follows that

$$
K_{3}=\frac{\eta}{2} Z_{\beta} .
$$

This automatically satisfies Eqn.(C28). With these redefinitions I get from Eqn.(C30) that

$$
\partial_{\mu} X_{h_{*}}^{a \mu}=Z_{\beta} \partial_{\mu} f^{a \mu} .
$$

The right hand side vanishes upon using $f^{a \mu}=\partial_{\nu} B^{a \mu \nu}$. Also, Eqn.(C35) can be written as

$$
-X_{\bar{\omega}_{*}}^{a \mu}+\frac{1}{\zeta} \partial^{\mu} X_{\bar{\alpha}}^{a}-\frac{1}{\zeta} Z_{\beta} \partial^{\mu} f^{\prime a}+Z_{\beta} \Delta_{R}^{a \mu}=0 .
$$

Using Eqs. (D5),(D8),(D10) and (D16), I can define some new functions and write

$$
\begin{aligned}
X_{h}^{a} & =Z_{\omega} f^{a}+\bar{X}_{h}^{a}, \quad X_{\bar{\alpha}}^{a}=Z_{\beta} f^{\prime a}+\bar{X}_{\bar{\alpha}}^{a}, \\
X_{\bar{\omega}_{*}}^{a \mu} & =Z_{\beta} \Delta^{a \mu}+\frac{1}{\zeta} \partial^{\mu} \bar{X}_{\bar{\alpha}}^{a}, \quad X_{h_{*}}^{a \mu}=Z_{\beta} f^{a \mu}+\frac{1}{\zeta} \partial^{\mu} \bar{X}_{h_{*}}^{a}, \quad s_{R} \bar{X}_{h_{*}}^{a}=\bar{X}_{\bar{\alpha}}^{a} .
\end{aligned}
$$


Then Eqn.(D15) implies, because $\bar{X}_{h_{*}}^{a}$ is a function of the fields and not an arbitrarily chosen function, that

$$
\bar{X}_{h_{*}}^{a}=0 \text {, and hence } \bar{X}_{\bar{\alpha}}^{a}=0 .
$$

Putting these into Eqn. C40), I get $\bar{X}_{h}^{a}=0$. Therefore, I can now write down the general form of the ghost sector of the theory as

$$
\begin{gathered}
\mathscr{L}_{g}=Z_{\omega} \bar{\omega}^{a} \Delta_{R}^{a}+Z_{\beta} \bar{\beta}^{a} \Delta_{R}^{\prime a}+Z_{\beta} \bar{\omega}_{\mu}^{a} \Delta_{R}^{a \mu}+Z_{\beta} \bar{\alpha}^{a} f^{\prime a}+Z_{\omega} h^{a} f^{a}+Z_{\beta} h_{\mu}^{a}\left(f^{a \mu}+\partial^{\mu} n^{a}\right) \\
-Z_{\beta} \partial^{\mu} \bar{\omega}_{\mu}^{a} \alpha^{a}+\zeta Z_{\beta} \bar{\alpha}^{a} \alpha^{a}+\frac{\xi}{2} Z_{\omega} h^{a} h^{a}+\frac{\eta}{2} Z_{\beta} h_{\mu}^{a} h^{a \mu} .
\end{gathered}
$$




\section{REFERENCES}

[1] G. 't Hooft, Nucl. Phys. B35, (1971) 167.

[2] A. Aurilia and Y. Takahashi, Prog. Theor. Phys. 66, (1981) 693.

[3] T. R. Govindarajan, J. Phys. G 8, (1982) L17.

[4] T. J. Allen, M. J. Bowick and A. Lahiri, Mod. Phys. Lett. A6, (1991) 559.

[5] J. A. Minahan and R. C. Warner, Stückelberg Revisited, Florida U. Preprint UFIFTHEP-89-15.

[6] T. Hurth, Helv. Phys. Acta 70, (1997) 406, hep-th/9511176.

[7] M. Henneaux et al, Phys. Lett. B410, (1997) 195, hep-th/9707129.

[8] A. Lahiri, Generating vector boson masses, Los Alamos report no. LA-UR-92-3477, hep-th/9301060.

[9] D. S. Hwang and C. -Y. Lee, J. Math. Phys. 38, 30 (1997), hep-th/9512216.

[10] A. Lahiri, Phys. Rev. D55, (1997) 5045, hep-ph/9609510.

[11] J. Barcelos-Neto and S. Rabello, Z.Phys. C74, (1997) 715-719, hep-th/9601076.

[12] J. Zinn-Justin, Quantum Field Theory and Critical Phenomena, Clarendon Press, Oxford, 1989.

[13] S. Weinberg, The Quantum Theory of Fields, Vol. 2: Modern Applications, Cambridge University Press, 1996.

[14] O. Piguet and S. P. Sorella, Algebraic Renormalization, Springer Lecture Notes in Physics vol. 28, 1995.

[15] M. Henneaux and C. Teitelboim, Quantization of Gauge Systems, Princeton University Press, 1992.

[16] A. S. Cattaneo et al, Commun. Math. Phys. 197, (1998) 571, hep-th/9705123.

[17] N. S. Manton, J.Math.Phys. 40, (1999) 736, hep-th/9806077. 


\section{TABLES}

TABLE I. Mass dimensions and ghost numbers of the fields and their antisources.

\begin{tabular}{lcccc}
\hline \hline $\begin{array}{l}\text { Field } \\
\chi^{A}\end{array}$ & dimension & ghost number & $\begin{array}{c}\text { dimension } \\
\text { of } K^{A}\end{array}$ & $\begin{array}{c}\text { ghost number } \\
\text { of } K^{A}\end{array}$ \\
\hline$A_{\mu}^{a}$ & 1 & 0 & 2 & -1 \\
$B_{\mu \nu}^{a}$ & 1 & 0 & 2 & -1 \\
$C_{\mu}^{a}$ & 0 & 0 & 3 & -1 \\
$\omega^{a}$ & 1 & 1 & 2 & -2 \\
$\bar{\omega}^{a}$ & 1 & -1 & 2 & 0 \\
$h^{a}$ & 2 & 0 & $\star$ & $\star$ \\
$\omega^{a \mu}$ & 1 & 1 & 2 & -2 \\
$\bar{\omega}^{a \mu}$ & 1 & -1 & 2 & 0 \\
$\theta^{a}$ & 0 & 1 & 3 & -2 \\
$h^{a \mu}$ & 2 & 0 & $\star$ & $\star$ \\
$n^{a}$ & 1 & 0 & 2 & -1 \\
$\beta^{a}$ & 1 & 2 & 2 & -3 \\
$\bar{\beta}^{a}$ & 1 & -2 & 2 & $\star$ \\
$\alpha^{a}$ & 2 & 1 & $\star$ & $\star$ \\
$\bar{\alpha}^{a}$ & 2 & -1 & $\star$ & $\star$ \\
$\alpha$ & 0 & 1 & $\star$ & $\star$ \\
\hline \hline
\end{tabular}

$\mathrm{A} \star$ indicates that the antisource $K^{A}$ does not appear in the theory as the BRST variation of the corresponding field $\chi^{A}$ vanishes. 